Saclay IPhT-T12/161 UUITP-22/12

\title{
Two-Loop Maximal Unitarity with External Masses
}

\author{
Henrik Johansson, ${ }^{1}$ David A. Kosower, ${ }^{1}$ and Kasper J. Larsen ${ }^{1,2}$ \\ ${ }^{1}$ Institut de Physique Théorique, CEA-Saclay, \\ F-91191 Gif-sur-Yvette cedex, France \\ Henrik. Johansson@cea.fr \\ David.Kosower@cea.fr \\ ${ }^{2}$ Department of Physics and Astronomy, \\ Uppsala University, SE-75108 Uppsala, Sweden \\ Kasper.Larsen@cea.fr
}

\begin{abstract}
We extend the maximal unitarity method at two loops to double-box basis integrals with up to three external massive legs. We use consistency equations based on the requirement that integrals of total derivatives vanish. We obtain unique formulæ for the coefficients of the master double-box integrals. These formulæ can be used either analytically or numerically.

PACS numbers: 11.15.-q, 11.15.Bt, 11.55.Bq, 12.38.-t, 12.38.Bx
\end{abstract}




\section{INTRODUCTION}

A quantitative understanding of backgrounds at the LHC to new-physics signals is important to direct searches for physics beyond the Standard Model. A similar understanding of signals of heavy Standard-Model particles is important to detailed studies of their properties as another window into new physics. Such quantitative understanding requires at least next-to-leading order (NLO) calculations in quantum chromodynamics (QCD). These calculations make use of a number of ingredients beyond the tree-level amplitudes required for a leading-order (LO) calculation: real-emission corrections, with an additional emitted gluon, or a gluon splitting into a quark-antiquark pair; and virtual one-loop corrections, with a virtual gluon or virtual quark in a closed loop. The required one-loop corrections are challenging with traditional Feynman-diagram methods, and become considerably more difficult as the number of final-state partons (gluons or quarks) grows.

Some subprocesses are absent at tree level, such as the gluon fusion to diphoton subprocess, $g g \rightarrow \gamma \gamma$, which is an important background to measurements of the recently-discovered new heavy boson (NHB) at the LHC [1, 2]. While these subprocesses are nominally of higher order in the strong coupling $\alpha_{s}$, the large gluon parton density at smaller $x$ can compensate for this additional power, giving rise to contributions to cross sections which are comparable to those from tree-level quark-initiated subprocesses [3-5]. The production of electroweak boson pairs, $g g \rightarrow Z \gamma, Z Z, W^{+} W^{-}$also falls into this class. Because the basic processes only arise from one-loop amplitudes, computation of the NLO corrections requires two-loop amplitudes [6] (as well as one-loop amplitudes with an additional parton, and singular factors derived from one-loop amplitudes).

Two-loop amplitudes are also required for any studies beyond NLO. Next-to-next-toleading order (NNLO) fixed-order calculations stand at the next frontier of precision QCD calculations. The only existing fully-exclusive NNLO jet calculations to date are for three-

jet production in electron-positron annihilation [7]. These are necessary to determine $\alpha_{s}$ to $1 \%$ accuracy from jet data at LEP [8], competitively with other determinations. At the LHC, NNLO calculations will be useful for determining an honest theoretical uncertainty estimate on NLO calculations, for assessing scale stability in multi-scale processes such as $W+$ multi-jet production, and will also be required for precision measurements of the NHB or of new physics should it be discovered. 
The unitarity method [9-25] has made many previously-inaccessible one-loop calculations feasible. Of particular note are processes with many partons in the final state. The most recent development, applying generalized unitarity, allows the method to be applied either analytically or purely numerically [26-36]. The numerical formalisms underly recent software libraries and programs that have been applied to LHC phenomenology. In this approach, the one-loop amplitude in QCD is written as a sum over a set of basis integrals, with coefficients that are rational in external spinors,

$$
\text { Amplitude }=\sum_{j \in \text { Basis }} \text { coefficient }_{j} \times \text { Integral }_{j}+\text { Rational }
$$

The integral basis for amplitudes with massless internal lines contains box, triangle, and bubble integrals in addition to purely rational terms (dropping all terms of $\mathcal{O}(\epsilon)$ in the dimensional regulator). The coefficients are calculated from products of tree amplitudes, typically by performing contour integrals via discrete Fourier projection.

The unitarity method has also been applied to higher-loop amplitudes. Some prior applications made use of 'minimal' generalized unitarity, cutting just enough propagators to break apart a higher-loop amplitude into a product of tree amplitudes. Each cut is again a product of tree amplitudes, but because not all possible propagators are cut, each generalized cut will correspond to several integrals, and algebra will be required to isolate specific integrals and their coefficients. This approach does not require a predetermined general basis of integrals. A number of calculations have been done this way, primarily in the $\mathcal{N}=4$ supersymmetric gauge theory [37-46], but including several four-point calculations in QCD and supersymmetric theories with less-than-maximal supersymmetry [55 61]. Furthermore, a number of recent multi-loop calculations in maximally supersymmetric gauge and gravity theories have used maximal cuts [47 54], without complete localization of integrands.

In maximal generalized unitarity as we pursue it, one cuts as many propagators as possible, and seeks to fully localize integrands onto global poles. In principle, this allows one to isolate individual integrals on the right-hand side of the higher-loop analog of eq. (1.1). In a previous paper [62], two of the present authors showed how to extract the coefficients of the two master double boxes using a multi-dimensional contour around global poles. Each global pole corresponds to cutting all propagators, and in addition seeking the poles of remaining degrees of freedom (typically generated by the Jacobian from cutting the propagators). This approach may be viewed as a generalization to two loops of the work of Britto, Cachazo, and 
Feng [13], and of Forde [20]. In parallel, Mastrolia and Ossola [63] and Badger, Frellesvig, and Zhang [64] have showed how to generalize the approach of Ossola, Papadopoulos, and Pittau [18] to higher loops. We refer to refs. 65 69] for further recent developments within this approach. A recent paper by Zhang [70] adds tools required in such an approach for reducing integrands to a basis of monomials.

In this paper, we continue the maximal-unitarity approach of ref. [62], taking advantage of recent work [71] by Caron-Huot and one of the present authors showing that the set of distinct global poles is smaller than previously assumed. Higher-loop amplitudes can be written in a similar form to those at one loop (1.1), as a sum over an integral basis [72], along with possible rational terms. (At higher loops, the coefficients of the basis integrals are no longer rational functions of the external spinors alone, but will depend explicitly on the dimensional regulator $\epsilon$.) Furthermore, at two and higher loops, the number of master integrals for a given topology may depend on the number and arrangement of external masses [72].

As in ref. [62], we use the equations relating generic tensor integrals to basis (or master) integrals in order to ensure the consistency and completeness of the choice of contours. We obtain a set of unique projectors (or master contours) to compute the coefficients of double boxes with one, two or three external masses, one projector for each different master integral. We again work only to leading order in $\epsilon$ in the coefficients.

The extraction of the double-box coefficient bears a superficial similarity to the procedure that would be followed in the leading-singularity approach [49, 73], but unlike the latter, manifestly ensures the consistency of the extraction with respect to terms that vanish after integration. Such terms inevitably arise when using the integration-by-parts (IBP) approach [74 81] in relating formally-irreducible tensor integrals to basis integrals. The extraction of higher-order terms in $\epsilon$ or the coefficients of integrals with fewer propagators, both of which we leave to future work, would also be different.

In this paper, we will focus on extracting the coefficients of two-loop master integrals with massless internal lines. One can consider the generalization to the case with massive internal lines. So long as there are some massless internal lines, so that there is at least one chiral vertex, the integrand will still have global poles, and we expect the approach described here to generalize smoothly. Masses on internal lines are also related to the generalization to $D$-dimensional maximal unitarity, which we again leave to future work. 
In section [II, we present our parametrization of the loop momenta. In section III, we discuss the solutions to the heptacut equations, and in section IV we list the global poles of the double boxes. We discuss the constraint equations in section $\mathrm{V}$, and obtain our main results for the projectors extracting integral coefficients in section VI. Section VII gives examples of applying the technique described here, and provides cross-checks with known results. We give our conclusions in section VIII,

\section{PARAMETRIZING THE INTEGRAND}

It will be convenient for our purposes to adopt a different parametrization for the loop momenta from the one used in ref. [62]. The parametrization used here has the virtue of keeping all expressions manifestly rational throughout the derivation. In this parametrization, we will need spinors corresponding to massive legs. To define appropriate momenta, we make use of 'mutually projected' momenta as in the work of Ossola, Papadopoulos and Pittau [18] and Forde [20] on the extraction of triangle and bubble coefficients at one loop. We use $\left(k_{1}, k_{2}\right)$ and $\left(k_{3}, k_{4}\right)$ as mutually-projecting pairs, defining within each pair,

$$
\begin{aligned}
& k_{j, 1}^{b, \mu}=k_{j, 1}^{\mu}-\frac{k_{j, 1}^{2}}{2 k_{j, 1} \cdot k_{j, 2}^{b}} k_{j, 2}^{b, \mu}, \\
& k_{j, 2}^{b, \mu}=k_{j, 2}^{\mu}-\frac{k_{j, 2}^{2}}{2 k_{j, 2} \cdot k_{j, 1}^{b}} k_{j, 1}^{b, \mu} .
\end{aligned}
$$

By construction, $k_{j, 1}^{b}$ and $k_{j, 2}^{b}$ are massless momenta.

Define

$$
\rho_{j, i} \equiv \frac{k_{j, i}^{2}}{2 k_{j, i} \cdot k_{j, \bar{\imath}}},
$$

where $i$ denotes one momentum in the pair, and $\bar{\imath}$ the other.

We note that

$$
k_{j, 1} \cdot k_{j, 2}^{b}=k_{j, 1}^{b} \cdot k_{j, 2}=k_{j, 1}^{b} \cdot k_{j, 2}^{b} .
$$

Define

$$
\gamma_{j, 12} \equiv 2 k_{j, 1}^{b} \cdot k_{j, 2}^{b}
$$

we can solve for it to obtain,

$$
\gamma_{j, 12}^{ \pm}=k_{j, 1} \cdot k_{j, 2} \pm\left[\left(k_{j, 1} \cdot k_{j, 2}\right)^{2}-k_{j, 1}^{2} k_{j, 2}^{2}\right]^{1 / 2}
$$


If either momentum $k_{j, i}$ is massless, only one solution survives, $\gamma_{j, 12}=2 k_{j, 1} \cdot k_{j, 2}$.

With two mutually-projecting pairs, we may have two independent $\gamma_{\mathrm{s}}, \gamma_{12}$ for the $\left(k_{1}, k_{2}\right)$ pair, and $\gamma_{34}$ for the $\left(k_{3}, k_{4}\right)$ pair. In general, we expect to express our final results in terms of the independent invariants $s_{12}$ and $s_{14}$, the nonzero masses amongst $\left\{m_{1}, m_{2}, m_{3}, m_{4}\right\}$, along with $\gamma_{12}$ (if $m_{1} \neq 0 \neq m_{2}$ ), and $\gamma_{34}$ (if $m_{3} \neq 0 \neq m_{4}$ ).

Inverting eq. (2.1) we obtain the massless momenta

$$
k_{j, i}^{b, \mu}=\left(1-\rho_{j, 1} \rho_{j, 2}\right)^{-1}\left(k_{j, i}^{\mu}-\rho_{j, i} k_{j, \bar{n}}^{\mu}\right) .
$$

We adopt the following parametrization for the on-shell double-box loop momenta,

$$
\begin{aligned}
& \ell_{1}^{\mu}=\frac{1}{2}\left\langle\lambda_{1}^{-}|\mu| \tilde{\lambda}_{1}^{\prime-}\right\rangle=\frac{1}{2}\left\langle\lambda_{1}|\mu| \tilde{\lambda}_{1}^{\prime}\right], \\
& \ell_{2}^{\mu}=\frac{1}{2}\left\langle\lambda_{2}^{-}|\mu| \tilde{\lambda}_{2}^{\prime-}\right\rangle=\frac{1}{2}\left\langle\lambda_{2}|\mu| \tilde{\lambda}_{2}^{\prime}\right] .
\end{aligned}
$$

This form ensures that $\ell_{1}^{2}=0=\ell_{2}^{2}$. We write the various loop spinors in terms of the spinors corresponding to $\left(k_{1}^{b}, k_{2}^{b}\right)$ for $\ell_{1}$, and the spinors corresponding to $\left(k_{3}^{b}, k_{4}^{b}\right)$ for $\ell_{2}$ :

$$
\begin{aligned}
& \left|\lambda_{1}{ }^{+}\right\rangle=\xi_{1}\left|1^{b,+}\right\rangle+\xi_{2} \frac{\left\langle 4^{b} 1^{b}\right\rangle}{\left\langle 4^{b} 2^{b}\right\rangle}\left|2^{b,+}\right\rangle, \\
& \left|\tilde{\lambda}^{\prime}{ }^{-}\right\rangle=\xi_{1}^{\prime}\left|1^{b,-}\right\rangle+\xi_{2}^{\prime} \frac{\left[4^{b} 1^{b}\right]}{\left[4^{b} 2^{b}\right]}\left|2^{b,-}\right\rangle, \\
& \left|\lambda_{2}{ }^{+}\right\rangle=\xi_{3} \frac{\left\langle 1^{b} 4^{b}\right\rangle}{\left\langle 1^{b} 3^{b}\right\rangle}\left|3^{b,+}\right\rangle+\xi_{4}\left|4^{b,+}\right\rangle, \\
& \left|\tilde{\lambda}^{\prime}{ }^{-}\right\rangle=\xi_{3}^{\prime} \frac{\left[1^{b} 4^{b}\right]}{\left[1^{b} 3^{b}\right]}\left|3^{b,-}\right\rangle+\xi_{4}^{\prime}\left|4^{b,-}\right\rangle .
\end{aligned}
$$

Without loss of generality, we can set $\xi_{1}=1=\xi_{4}$. We will use the simplified notation $|j\rangle \equiv\left|j^{+}\right\rangle$and $\left.\mid j\right] \equiv\left|j^{-}\right\rangle$in later sections of the paper.

\section{HEPTACUT EQUATIONS}

We focus on the planar double-box integral, shown in fig. 1, which is given by,

$$
P_{2,2}^{* *}=\int \frac{d^{D} \ell_{1}}{(2 \pi)^{D}} \frac{d^{D} \ell_{2}}{(2 \pi)^{D}} \frac{1}{\ell_{1}^{2}\left(\ell_{1}-k_{1}\right)^{2}\left(\ell_{1}-K_{12}\right)^{2}\left(\ell_{1}+\ell_{2}\right)^{2} \ell_{2}^{2}\left(\ell_{2}-k_{4}\right)^{2}\left(\ell_{2}-K_{34}\right)^{2}},
$$

where $K_{i \cdots j} \equiv k_{i}+\cdots+k_{j}$, and the notation follows ref. [72]. We allow up to three of the external legs to be massive, leaving the four-mass case for future investigation. We leave the Feynman $i \varepsilon$ prescription implicit. 


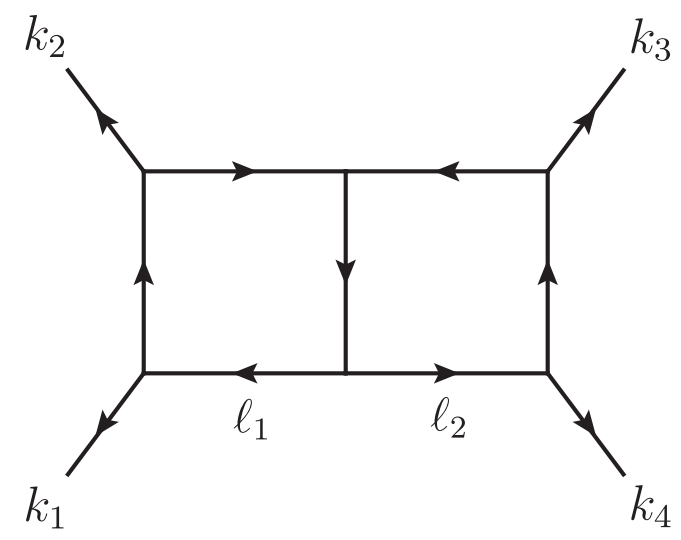

FIG. 1: The double-box integral $P_{2,2}^{* *}$.

The heptacut equations correspond to cutting all seven propagators, that is setting all denominator factors in eq. (3.1) to zero simultaneously. Imposing the heptacut can be implemented by replacing the original contour of integration for the loop momenta, running along the real axis for each component of $\ell_{1,2}^{\mu}$, by a seven-torus encircling the solution surface(s) for the vanishing of these denominator factors. Two of these equations are already solved by the parametrization in eq. (2.7). Using this parametrization, the remaining heptacut equations involving only $\ell_{1}$ take the form,

$$
\begin{aligned}
\left(\ell_{1}-k_{1}\right)^{2} & =0 \Rightarrow \\
m_{1}^{2} & =\left(\rho_{1,1} \xi_{1}^{\prime}+\frac{k_{1}^{b} \cdot k_{4}^{b}}{k_{2}^{b} \cdot k_{4}^{b}} \xi_{2} \xi_{2}^{\prime}\right) \gamma_{12}, \\
\left(\ell_{1}-K_{12}\right)^{2} & =0 \Rightarrow \\
s_{12}-m_{1}^{2} & =\left(\xi_{1}^{\prime}+\rho_{1,2} \frac{k_{1}^{b} \cdot k_{4}^{b}}{k_{2}^{b} \cdot k_{4}^{b}} \xi_{2} \xi_{2}^{\prime}\right) \gamma_{12} .
\end{aligned}
$$

In the general case $\left(m_{1} \neq 0 \neq m_{2}\right)$, these equations have the solution,

$$
\begin{aligned}
& \xi_{1}^{\prime}=\frac{\gamma_{12} s_{12}-\left(\gamma_{12}+m_{2}^{2}\right) m_{1}^{2}}{\gamma_{12}^{2}-m_{1}^{2} m_{2}^{2}} \equiv \bar{\xi}_{1}^{\prime}, \\
& \xi_{2}^{\prime}=-\frac{m_{1}^{2}\left(s_{12}-\gamma_{12}-m_{1}^{2}\right) k_{2}^{b} \cdot k_{4}^{b}}{\xi_{2}\left(\gamma_{12}^{2}-m_{1}^{2} m_{2}^{2}\right) k_{1}^{b} \cdot k_{4}^{b}} \equiv \frac{\bar{\xi}_{2}^{\prime}}{\xi_{2}} .
\end{aligned}
$$

There are similar solutions for the equations involving only $\ell_{2}$.

The first of these solutions has the correct limits when either $m_{1} \rightarrow 0$ or $m_{2} \rightarrow 0$ (or both); the second solution, in contrast, is replaced by the pair of solutions,

$$
\xi_{2}=0, \quad \xi_{2}^{\prime} \text { free; } \quad \text { or } \quad \xi_{2}^{\prime}=0, \quad \xi_{2} \text { free }
$$


More explicitly, when $m_{1}=0$ but $m_{2} \neq 0$,

$$
\xi_{1}^{\prime}=\frac{s_{12}}{\gamma_{12}}=1+\rho_{1,2}
$$

when $m_{2}=0$ (whether $m_{1}$ vanishes or not),

$$
\xi_{1}^{\prime}=1
$$

The last heptacut equation is $\ell_{1} \cdot \ell_{2}=0$, which takes the form,

$$
\begin{aligned}
& 0=-\frac{1}{2}\left\langle 1^{b} 4^{b}\right\rangle\left[1^{b} 4^{b}\right]\left(1+\xi_{2}+\xi_{3}+\xi_{2} \xi_{3} \frac{\left\langle 1^{b} 4^{b}\right\rangle\left\langle 2^{b} 3^{b}\right\rangle}{\left\langle 2^{b} 4^{b}\right\rangle\left\langle 1^{b} 3^{b}\right\rangle}\right) \\
& \times\left(\bar{\xi}_{1}^{\prime} \bar{\xi}_{4}^{\prime}+\frac{\bar{\xi}_{2}^{\prime} \bar{\xi}_{4}^{\prime}}{\xi_{2}}+\frac{\bar{\xi}_{1}^{\prime} \bar{\xi}_{3}^{\prime}}{\xi_{3}}+\frac{\bar{\xi}_{2}^{\prime} \bar{\xi}_{3}^{\prime}}{\xi_{2} \xi_{3}} \frac{\left[1^{b} 4^{b}\right]\left[2^{b} 3^{b}\right]}{\left[2^{b} 4^{b}\right]\left[1^{b} 3^{b}\right]}\right) .
\end{aligned}
$$

This equation has two solutions corresponding to the two factors,

$$
\xi_{3}=\left\{\begin{array}{c}
-\frac{1+\xi_{2}}{1+\tau \xi_{2}}, \\
-\frac{\left(\xi_{2} \bar{\xi}_{1}^{\prime}+\bar{\tau}_{2}^{\prime}\right) \bar{\xi}_{3}^{\prime}}{\left(\xi_{2} \bar{\xi}_{1}^{\prime}+\bar{\xi}_{2}^{\prime}\right) \bar{\xi}_{4}^{\prime}}
\end{array}\right.
$$

where

$$
\begin{aligned}
\tau & \equiv \frac{\left\langle 1^{b} 4^{b}\right\rangle\left\langle 2^{b} 3^{b}\right\rangle}{\left\langle 2^{b} 4^{b}\right\rangle\left\langle 1^{b} 3^{b}\right\rangle} \\
\bar{\tau} & \equiv \frac{\left[1^{b} 4^{b}\right]\left[2^{b} 3^{b}\right]}{\left[2^{b} 4^{b}\right]\left[1^{b} 3^{b}\right]}
\end{aligned}
$$

In fact,

$$
\begin{aligned}
\tau=\bar{\tau}=- & \frac{\gamma_{34}\left(\gamma_{12}+m_{1}^{2}\right)}{\left(\gamma_{34}+m_{3}^{2}\right)\left[\left(\gamma_{12} \gamma_{34}-m_{1}^{2} m_{3}^{2}\right)\left(\gamma_{12} \gamma_{34}-m_{2}^{2} m_{4}^{2}\right)+\gamma_{12} \gamma_{34} s_{12} s_{14}\right]} \\
& \times\left[\left(\gamma_{12}+m_{2}^{2}\right)\left(\gamma_{34}+m_{3}^{2}\right)\left(m_{2}^{2}+m_{3}^{2}-s_{14}\right)+2 m_{2}^{2} m_{3}^{2} s_{12}\right. \\
& \left.\quad+\left(\gamma_{12}+m_{2}^{2}\right) m_{3}^{2}\left(m_{1}^{2}-m_{2}^{2}-s_{12}\right)+\left(\gamma_{34}+m_{3}^{2}\right) m_{2}^{2}\left(m_{4}^{2}-m_{3}^{2}-s_{12}\right)\right] .
\end{aligned}
$$

As in ref. [71], we must consider three different classes of solutions, corresponding to different configurations of external masses.

In considering massless limits, there are three classes to consider: (a) when neither pair $(1,2)$ or $(3,4)$ contains a massless momentum; (b) when exactly one pair contains a massless momentum; (c) when both pairs contain massless momenta. The first class corresponds to the four-mass double box; the second class, to the three-mass double box and the short-side 


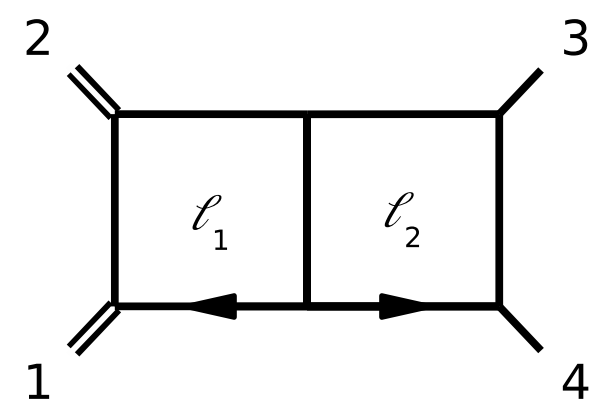

FIG. 2: The short-side two-mass double box. Single lines indicate massless legs, and doubled lines massive legs (here the two legs on the left-hand side).

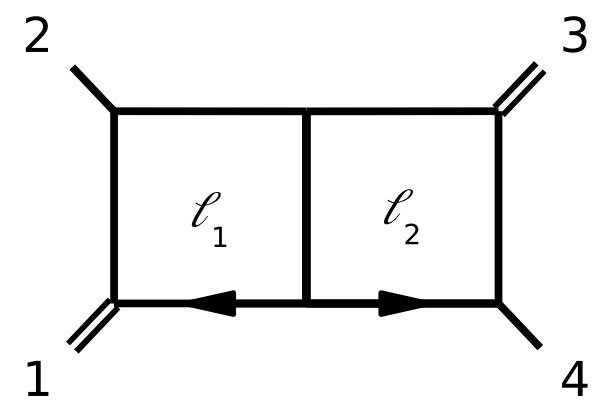

(i)

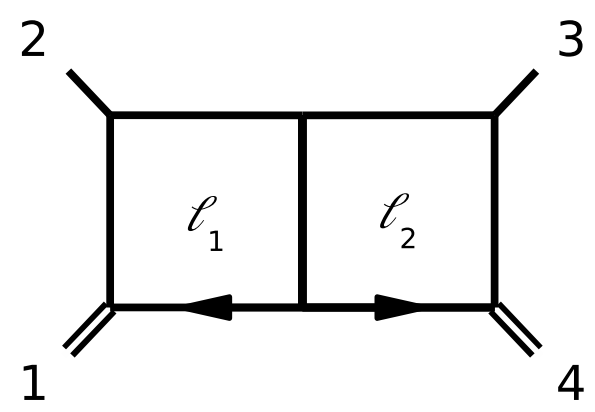

(ii)

FIG. 3: Other two-mass double-boxes: (i) the diagonal one (ii) the long-side one. Single lines indicate massless legs, and doubled lines massive legs (in (i), the lower-left and upper-right legs, and in (ii), the two lower legs).

two-mass one, shown in fig. 2, the last class, to the remaining configurations - diagonal and long-side two-mass double boxes, shown in fig. 3, along with the one-mass and massless ones. Class (a) is given by the solutions above, but we will not consider it any further in the present paper.

In class (b), it suffices to take the massless momentum to be either $k_{3}$ or $k_{4}$ (or both). There are two solutions to the $\ell_{2}$ equations to consider, $\xi_{3}=0$ and $\xi_{3}^{\prime}=0$. For the first, the last heptacut equation becomes,

$$
0=\left\langle 1^{b} 4^{b}\right\rangle\left[1^{b} 4^{b}\right]\left(1+\xi_{2}\right)\left(\bar{\xi}_{1}^{\prime} \bar{\xi}_{4}^{\prime}+\frac{\bar{\xi}_{2}^{\prime} \bar{\xi}_{4}^{\prime}}{\xi_{2}}+\bar{\xi}_{1}^{\prime} \xi_{3}^{\prime}+\bar{\tau} \frac{\bar{\xi}_{2}^{\prime} \xi_{3}^{\prime}}{\xi_{2}}\right)
$$

This equation again has two solutions, $\xi_{2}=-1$ (with $\xi_{3}^{\prime}$ being identified as the remaining 
variable $z$ ), and the other having,

$$
\xi_{3}^{\prime}=-\frac{\bar{\xi}_{4}^{\prime}\left(\bar{\xi}_{1}^{\prime} \xi_{2}+\bar{\xi}_{2}^{\prime}\right)}{\bar{\xi}_{1}^{\prime} \xi_{2}+\bar{\tau} \bar{\xi}_{2}^{\prime}}
$$

with $\xi_{2}=z$. The second solution to the $\ell_{2}$ heptacut equations has $\xi_{3}^{\prime}=0$, whereupon the last heptacut equation becomes,

$$
0=\left\langle 1^{b} 4^{b}\right\rangle\left[1^{b} 4^{b}\right]\left(1+\xi_{2}+\xi_{3}+\tau \xi_{2} \xi_{3}\right)\left(\bar{\xi}_{1}^{\prime} \bar{\xi}_{4}^{\prime}+\frac{\bar{\xi}_{2}^{\prime} \bar{\xi}_{4}^{\prime}}{\xi_{2}}\right),
$$

which also has two solutions,

$$
\xi_{2}=-\frac{\bar{\xi}_{2}^{\prime}}{\bar{\xi}_{1}^{\prime}}
$$

(with $\xi_{3}=z$ ), and

$$
\xi_{3}=-\frac{1+\xi_{2}}{1+\tau \xi_{2}}
$$

(with $\xi_{2}=z$ ). Overall in class (b), we have four solutions to the heptacut equations, in agreement with the discussion in ref. [71], with $\xi_{1}^{\prime}=\bar{\xi}_{1}^{\prime}, \xi_{4}^{\prime}=\bar{\xi}_{4}^{\prime}$ in all, and the four variables $\left(\xi_{2}, \xi_{2}^{\prime}, \xi_{3}, \xi_{3}^{\prime}\right)$ taking on the following values,

$$
\begin{aligned}
& \mathcal{S}_{1}:\left(-\frac{\bar{\xi}_{2}^{\prime}}{\bar{\xi}_{1}^{\prime}},-\bar{\xi}_{1}^{\prime}, z, 0\right), \\
& \mathcal{S}_{2}: \quad\left(z, \frac{\bar{\xi}_{2}^{\prime}}{z},-\frac{1+z}{1+\tau z}, 0\right), \\
& \mathcal{S}_{3}: \quad\left(-1,-\bar{\xi}_{2}^{\prime}, 0, z\right), \\
& \mathcal{S}_{4}: \quad\left(z, \frac{\bar{\xi}_{2}^{\prime}}{z}, 0,-\frac{\bar{\xi}_{4}^{\prime}\left(\bar{\xi}_{1}^{\prime} z+\bar{\xi}_{2}^{\prime}\right)}{\bar{\xi}_{1}^{\prime} z+\bar{\tau} \bar{\xi}_{2}^{\prime}}\right) .
\end{aligned}
$$

In class (c), we have four solutions to the pure- $\ell_{1,2}$ equations to consider,

$$
\begin{aligned}
& \xi_{2}=0 \text { and } \xi_{3}=0 \text {, } \\
& \xi_{2}=0 \text { and } \xi_{3}^{\prime}=0 \text {, } \\
& \xi_{2}^{\prime}=0 \text { and } \xi_{3}=0 \text {, } \\
& \xi_{2}^{\prime}=0 \text { and } \xi_{3}^{\prime}=0 \text {. }
\end{aligned}
$$

In the first of these solutions, the last heptacut equation simplifies to,

$$
0=\left\langle 1^{b} 4^{b}\right\rangle\left[1^{b} 4^{b}\right]\left(\bar{\xi}_{1}^{\prime} \bar{\xi}_{4}^{\prime}+\xi_{2}^{\prime} \bar{\xi}_{4}^{\prime}+\bar{\xi}_{1}^{\prime} \xi_{3}^{\prime}+\bar{\tau} \xi_{2}^{\prime} \xi_{3}^{\prime}\right),
$$

which has the solution,

$$
\xi_{3}^{\prime}=-\frac{\bar{\xi}_{4}^{\prime}\left(\bar{\xi}_{1}^{\prime}+\xi_{2}^{\prime}\right)}{\bar{\xi}_{1}^{\prime}+\bar{\tau} \xi_{2}^{\prime}}
$$


(with $\xi_{2}^{\prime}=z$ ). In the second solution in eq. (3.17), the last heptacut equation simplifies to,

$$
0=\left\langle 1^{b} 4^{b}\right\rangle\left[1^{b} 4^{b}\right]\left(1+\xi_{3}\right)\left(\bar{\xi}_{1}^{\prime} \bar{\xi}_{4}^{\prime}+\xi_{2}^{\prime} \bar{\xi}_{4}^{\prime}\right)
$$

which has two solutions, $\xi_{3}=-1$ (with $\left.\xi_{2}^{\prime}=z\right)$, and $\xi_{2}^{\prime}=-\bar{\xi}_{1}^{\prime}$ (with $\xi_{3}=z$ ).

In the third solution in eq. (3.17), the last heptacut equation simplifies to,

$$
0=\left\langle 1^{b} 4^{b}\right\rangle\left[1^{b} 4^{b}\right]\left(1+\xi_{2}\right)\left(\bar{\xi}_{1}^{\prime} \bar{\xi}_{4}^{\prime}+\bar{\xi}_{1}^{\prime} \xi_{3}^{\prime}\right)
$$

which also has two solutions, $\xi_{2}=-1$ (with $\xi_{3}^{\prime}=z$ ), and $\xi_{3}^{\prime}=-\bar{\xi}_{4}^{\prime}$ (with $\xi_{2}=z$ ).

In the last solution to eq. (3.17), the last heptacut equation simplifies to,

$$
0=\left\langle 1^{b} 4^{b}\right\rangle\left[1^{b} 4^{b}\right] \bar{\xi}_{1}^{\prime} \bar{\xi}_{4}^{\prime}\left(1+\xi_{2}+\xi_{3}+\tau \xi_{2} \xi_{3}\right)
$$

which has a single solution,

$$
\xi_{3}=-\frac{1+\xi_{2}}{1+\tau \xi_{2}}
$$

(with $\left.\xi_{2}=z\right)$.

Overall in class (c), we have six solutions to the heptacut equations, in agreement with refs. [62, 71], with $\xi_{1}^{\prime}=\bar{\xi}_{1}^{\prime}, \xi_{4}^{\prime}=\bar{\xi}_{4}^{\prime}$ in all, and the four variables $\left(\xi_{2}, \xi_{2}^{\prime}, \xi_{3}, \xi_{3}^{\prime}\right)$ taking on the following values,

$$
\begin{array}{ll}
\mathcal{S}_{1}: & \left(0,-\bar{\xi}_{1}^{\prime}, z, 0\right), \\
\mathcal{S}_{2}: & (0, z,-1,0), \\
\mathcal{S}_{3}: & (-1,0,0, z), \\
\mathcal{S}_{4}: & \left(z, 0,0,-\bar{\xi}_{4}^{\prime}\right), \\
\mathcal{S}_{5}: & \left(z, 0,-\frac{1+z}{1+\tau z}, 0\right), \\
\mathcal{S}_{6}: & \left(0, z, 0,-\frac{\bar{\xi}_{4}^{\prime}\left(\bar{\xi}_{1}^{\prime}+z\right)}{\bar{\xi}_{1}^{\prime}+\bar{\tau} z}\right) .
\end{array}
$$

\section{GLOBAL POLES}

As we take the cuts strictly in four dimensions, the two loop momenta have a total of eight degrees of freedom. After changing variables to the various $\xi_{i}$ and $\xi_{i}^{\prime}$, replacing the original contours of integration with tori encircling the locations of the zeros of the denominator of the integrand localizes it on the solutions of the heptacut equations. We found the solutions 
to these equations in the previous section. In the all-massless, one-mass, diagonal two-mass, and long-side two-mass double boxes, corresponding to class (c), there are six independent solutions. In the short-side two-mass and in the three-mass double boxes, corresponding to class (b), there are four independent solutions.

In either case, the heptacut equations fix only seven of the eight degrees of freedom. Performing the corresponding contour integrals gives rise to an inverse Jacobian from diagonalizing and linearizing the denominator in the neighborhood of the simultaneous zeros in each of the denominator factors. In all cases, this inverse Jacobian also has poles in the remaining degree of freedom $z$. In certain solutions, powers of one or the other of the loop momenta will also have poles in $z$. These poles, along with the Jacobian poles, make it possible to choose contours for the $z$ integration which further localizes the integral. As we shall see, this in turn makes it possible to distinguish between the various master integrals.

There are two distinct Jacobians that arise. One emerges from the change of variables $\ell_{1,2}^{\mu}$ to the $\xi$ parameters. This Jacobian appears in the numerator. The other is the inverse Jacobian that arises from performing the heptacut contour integrals. Because the parametrization in eqs. (2.7)-(2.8) automatically ensures that $\ell_{1}^{2}=0=\ell_{2}^{2}$, it is not suitable for computing these Jacobians. As a remedy, add an additional vector to the original parametrization (2.7),

$$
\begin{aligned}
\ell_{1}^{\mu} & =\frac{1}{2}\left\langle\lambda_{1}^{-}|\mu| \tilde{\lambda}_{1}^{\prime-}\right\rangle+\zeta_{1} \eta_{1}^{\mu}, \\
\ell_{2}^{\mu} & =\frac{1}{2}\left\langle\lambda_{2}^{-}|\mu| \tilde{\lambda}_{2}^{\prime-}\right\rangle+\zeta_{2} \eta_{2}^{\mu},
\end{aligned}
$$

where $\zeta_{i}$ are complex numbers, and the $\eta_{i}$ are null vectors satisfying $\eta_{1}\left|\lambda_{1}{ }^{+}\right\rangle \neq 0 \neq \eta_{1}\left|\tilde{\lambda}_{1}^{\prime}{ }^{-}\right\rangle$ and $\not_{2}\left|\lambda_{2}{ }^{+}\right\rangle \neq 0 \neq \not_{2}\left|\tilde{\lambda}_{2}^{\prime}{ }^{-}\right\rangle$.

In both class (b) and (c), we may choose $\eta_{1}=k_{2}^{b}$ and $\eta_{2}=k_{3}^{b}$. This is a suitable choice so long as $\xi_{1}^{\prime} \neq 0$, so that the non-collinearity condition above is satisfied. For generic momenta, this holds. With the additional term, the equations $\ell_{1}^{2}=0=\ell_{2}^{2}$ become,

$$
\begin{aligned}
& 0=\gamma_{12} \xi_{1}^{\prime} \zeta_{1}, \\
& 0=\gamma_{34} \xi_{4}^{\prime} \zeta_{2} .
\end{aligned}
$$

The requirement that $\xi_{1}^{\prime} \neq 0 \neq \xi_{4}^{\prime}$ leaves only the obvious solutions, $\zeta_{1}=0$ and $\zeta_{2}=0$, respectively, to these equations. 
The Jacobian for the change of variables is,

$$
J_{j}=\operatorname{det}_{\mu, i} \frac{\partial \ell_{j}^{\mu}}{\partial v_{j, i}},
$$

where $v_{j, 1}=\zeta_{j}, v_{1,2}=\xi_{1}^{\prime}, v_{2,2}=\xi_{4}^{\prime}, v_{1,3}=\xi_{2}, v_{2,3}=\xi_{3}, v_{1,4}=\xi_{2}^{\prime}$, and $v_{2,4}=\xi_{3}^{\prime}$.

In all solutions in both classes (b) and (c), these Jacobians take the form,

$$
\begin{aligned}
& J_{1}=i \frac{\gamma_{12}^{2} k_{1}^{b} \cdot k_{4}^{b} \xi_{1}^{\prime}}{4\left(k_{2}^{b} \cdot k_{4}^{b}\right)} \\
& J_{2}=i \frac{\gamma_{34}^{2} k_{1}^{b} \cdot k_{4}^{b} \xi_{4}^{\prime}}{4\left(k_{1}^{b} \cdot k_{3}^{b}\right)} .
\end{aligned}
$$

The Jacobian that arises from performing the heptacut contour integrals (which will appear in the denominator) is,

$$
J_{\mathrm{h}}=\operatorname{det}_{i, j} \frac{\partial P_{i}}{\partial w_{j}}
$$

where $P_{i}$ is the $i$-th propagator denominator, and the set $\left\{w_{j}\right\}$ are the seven variables (out of the eight $\left.\xi_{1}^{\prime}, \xi_{2}, \xi_{2}^{\prime}, \xi_{3}, \xi_{3}^{\prime}, \xi_{4}^{\prime}, \zeta_{1}, \zeta_{2}\right)$ frozen by the contour integrations.

This Jacobian is not the same for all solutions $\mathcal{S}_{i}$. As discussed earlier, it will appear in the denominator; the overall Jacobian appearing after performing the heptacut contour integrals is,

$$
J_{\oint}^{(0)}=\frac{J_{1} J_{2}}{J_{\mathrm{h}}}
$$

For our purposes, it will be convenient to factor out an overall prefactor which would otherwise cancel in final formulæ for integral coefficients, defining the reduced Jacobian for class (b) as,

$$
J_{\oint}=J_{\oint}^{(0)} \frac{\left(\bar{\xi}_{1}^{\prime}-\bar{\xi}_{2}^{\prime}\right) \bar{\xi}_{4}^{\prime}}{\gamma_{*}},
$$

and for class (c) as,

$$
J_{\oint}=J_{\oint}^{(0)} \frac{\bar{\xi}_{1}^{\prime} \bar{\xi}_{4}^{\prime}}{\gamma_{*}}
$$

where

$$
\gamma_{*} \equiv \frac{\gamma_{12} \gamma_{34}}{32 k_{1}^{b} \cdot k_{4}^{b}\left(\gamma_{12}^{2}-m_{1}^{2} m_{2}^{2}\right)\left(\gamma_{34}^{2}-m_{3}^{2} m_{4}^{2}\right)}
$$




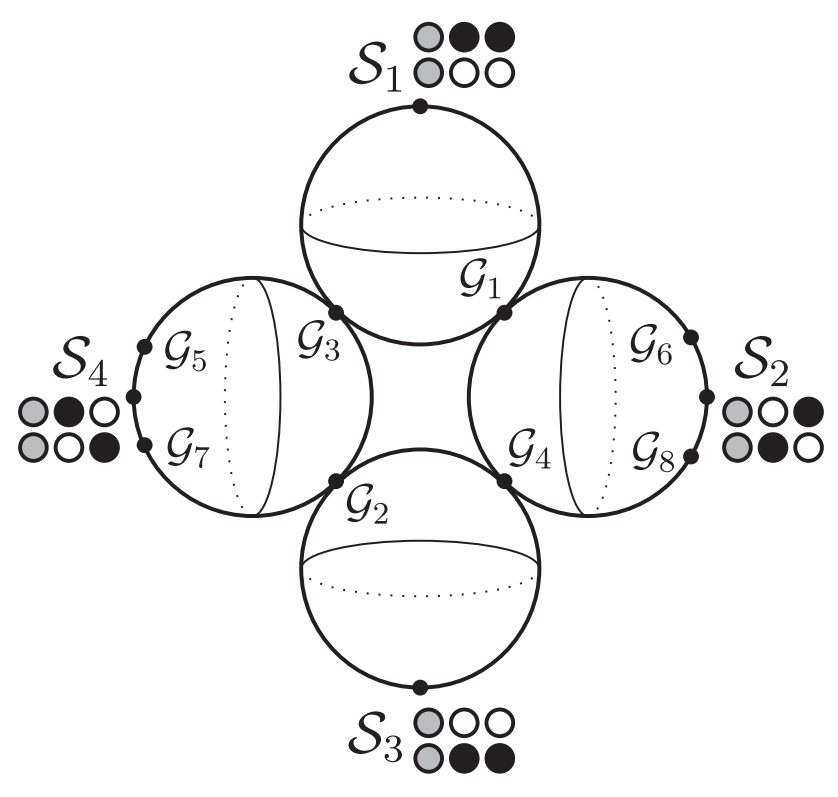

FIG. 4: A representation of the solution space for the class (b) heptacut equations, showing the four independent solutions $\mathcal{S}_{i}$, and the locations of the eight global poles $\mathcal{G}_{j}$. The small white, black and gray blobs indicate the pattern of chiral, antichiral and nonchiral kinematics, respectively, at the vertices of a double-box heptacut.

Within class (b), we then find,

$$
J_{\oint}= \begin{cases}J_{\oint}^{(\mathrm{b}, 1)} \equiv-\frac{\bar{\xi}_{1}^{\prime}-\bar{\xi}_{2}^{\prime}}{z\left(-\tau z \bar{\xi}_{2}^{\prime}+z \bar{\xi}_{1}^{\prime}+\bar{\xi}_{1}^{\prime}-\bar{\xi}_{2}^{\prime}\right)}, & \text { for solution } \mathcal{S}_{1} \\ J_{\oint}^{(\mathrm{b}, 2)} \equiv-\frac{\bar{\xi}_{1}^{\prime}-\bar{\xi}_{2}^{\prime}}{(z+1)\left(z \bar{\xi}_{1}^{\prime}+\bar{\xi}_{2}^{\prime}\right)}, & \text { for solution } \mathcal{S}_{2} \\ J_{\oint}^{(\mathrm{b}, 3)} \equiv-\frac{\left(\bar{\xi}_{1}^{\prime}-\bar{\xi}_{2}^{\prime}\right) \bar{\xi}_{4}^{\prime}}{z\left(-\bar{\tau} z \bar{\xi}_{2}^{\prime}+z \bar{\xi}_{1}^{\prime}+\bar{\xi}_{4}^{\prime} \bar{\xi}_{1}^{\prime}-\bar{\xi}_{2}^{\prime} \bar{\xi}_{4}^{\prime}\right)}, & \text { for solution } \mathcal{S}_{3} \\ J_{\oint}^{(\mathrm{b}, 4)} \equiv \frac{\bar{\xi}_{1}^{\prime}-\bar{\xi}_{2}^{\prime}}{(z+1)\left(z \bar{\xi}_{1}^{\prime}+\bar{\xi}_{2}^{\prime}\right)}, & \text { for solution } \mathcal{S}_{4} .\end{cases}
$$

The first of these Jacobians has poles at,

$$
\begin{aligned}
& z=0 \\
& z=-\frac{\bar{\xi}_{1}^{\prime}-\bar{\xi}_{2}^{\prime}}{\bar{\xi}_{1}^{\prime}-\tau \bar{\xi}_{2}^{\prime}}
\end{aligned}
$$

the second and fourth at,

$$
\begin{aligned}
& z=-1, \\
& z=-\frac{\bar{\xi}_{2}^{\prime}}{\bar{\xi}_{1}^{\prime}}
\end{aligned}
$$


and the third at,

$$
\begin{aligned}
& z=0, \\
& z=-\frac{\bar{\xi}_{4}^{\prime}\left(\bar{\xi}_{1}^{\prime}-\bar{\xi}_{2}^{\prime}\right)}{\bar{\xi}_{1}^{\prime}-\bar{\tau} \bar{\xi}_{2}^{\prime}} .
\end{aligned}
$$

Integrals with powers of the loop momentum in the numerator will have powers of the various coefficients $\xi_{2}, \xi_{2}^{\prime}, \xi_{3}, \xi_{3}^{\prime}$ once re-expressed in terms of our parametrization of loop momenta (2.7). Some of these coefficients also have poles in $z$, which leads to additional poles in the integrand beyond those in the Jacobian above. In each solution, there is a pole at $z=\infty$. From eq. (3.16), we see that there are further poles at,

$$
\begin{aligned}
& z=0, \\
& z=-\frac{1}{\tau},
\end{aligned}
$$

for solution $\mathcal{S}_{2}$, and,

$$
\begin{aligned}
& z=0, \\
& z=-\frac{\bar{\tau} \bar{\xi}_{2}^{\prime}}{\bar{\xi}_{1}^{\prime}},
\end{aligned}
$$

for solution $\mathcal{S}_{4}$.

Poles in $z$ complete the poles in the other seven degrees of freedom to provide a global pole. Ultimately, we will be interested in residues at these global poles. Not all poles within each solution are independent because the sum of residues vanishes, and so we can remove one pole within each solution $\mathcal{S}_{i}$. Furthermore, one might expect poles in $z$ in different solutions $\mathcal{S}_{i}$ to yield distinct global poles; but this is not the case. As pointed out [71] by Caron-Huot and one of the present authors, global poles within one solution may in fact end up being identical to global poles in another, in the sense of being located at the same values of $\ell_{1,2}$. After removing such duplicates, one finds a total of eight global poles. At all eight, $\xi_{1}^{\prime}=\bar{\xi}_{1}^{\prime}, \xi_{4}^{\prime}=\bar{\xi}_{4}^{\prime}$, and $\zeta_{1}=0=\zeta_{2}$, while the values of $\left(\xi_{2}, \xi_{2}^{\prime}, \xi_{3}, \xi_{3}^{\prime}\right)$ are as follows,

$$
\begin{aligned}
& \mathcal{G}_{1}: \quad\left(-\frac{\bar{\xi}_{2}^{\prime}}{\bar{\xi}_{1}^{\prime}},-\bar{\xi}_{1}^{\prime}, \frac{\bar{\xi}_{2}^{\prime}-\bar{\xi}_{1}^{\prime}}{\left.\bar{\xi}_{1}^{\prime}-\tau \bar{\xi}_{2}^{\prime}, 0\right),}\right. \\
& \mathcal{G}_{2}: \quad\left(-1,-\bar{\xi}_{2}^{\prime}, 0,-\frac{\left(\bar{\xi}_{1}^{\prime}-\bar{\xi}_{2}^{\prime}\right) \bar{\xi}_{4}^{\prime}}{\bar{\xi}_{1}^{\prime}-\bar{\tau} \bar{\xi}_{2}^{\prime}}\right), \\
& \mathcal{G}_{3}: \quad\left(-\frac{\bar{\xi}_{2}^{\prime}}{\bar{\xi}_{1}^{\prime}},-\bar{\xi}_{1}^{\prime}, 0,0\right),
\end{aligned}
$$




$$
\begin{aligned}
\mathcal{G}_{4}: & \left(-1,-\bar{\xi}_{2}^{\prime}, 0,0\right), \\
\mathcal{G}_{5}: & \left(0, \infty, 0,-\frac{\bar{\xi}_{4}^{\prime}}{\bar{\tau}}\right), \\
\mathcal{G}_{6}: & \left(\infty, 0,-\frac{1}{\tau}, 0\right), \\
\mathcal{G}_{7}: & \left(-\frac{\bar{\tau} \bar{\xi}_{2}^{\prime}}{\bar{\xi}_{1}^{\prime}},-\frac{\bar{\xi}_{1}^{\prime}}{\bar{\tau}}, 0, \infty\right), \\
\mathcal{G}_{8}: & \left(-\frac{1}{\tau},-\tau \bar{\xi}_{2}^{\prime}, \infty, 0\right) .
\end{aligned}
$$

This corresponds to picking the two additional poles in eq. (4.15) for solution $\mathcal{S}_{4}$, and the poles at $z=-1 / \tau$ and $z=\infty$ for solution $\mathcal{S}_{2}$.

We obtain the same poles whether we pick $\gamma_{12}=\gamma_{12}^{+}$or $\gamma_{12}^{-}$, so we can choose either. In future numerical applications, it may be more appropriate to average over the two choices.

In the poles where one parameter or another goes to infinity, we must specify the order of limits carefully.

In global pole $\mathcal{G}_{5}$, we should set,

$$
\xi_{2}^{\prime}=\frac{\bar{\xi}_{2}^{\prime}}{z}, \xi_{3}=0, \xi_{3}^{\prime}=-\frac{\bar{\xi}_{4}^{\prime}\left(z \bar{\xi}_{1}^{\prime}+\bar{\xi}_{2}^{\prime}\right)}{\bar{\tau} \bar{\xi}_{2}^{\prime}+z \bar{\xi}_{1}^{\prime}}, \xi_{2}=z,
$$

followed by the limit $z \rightarrow 0$; in global pole $\mathcal{G}_{6}$, we should set,

$$
\xi_{2}^{\prime}=\frac{\bar{\xi}_{2}^{\prime}}{z}, \xi_{3}^{\prime}=0, \xi_{3}=-\frac{z+1}{\tau z+1}, \xi_{2}=z,
$$

followed by the limit $z \rightarrow \infty$; in global pole $\mathcal{G}_{7}$, we should set,

$$
\xi_{2}^{\prime}=\frac{\bar{\xi}_{2}^{\prime}}{z}, \xi_{3}=0, \xi_{3}^{\prime}=-\frac{\bar{\xi}_{4}^{\prime}\left(z \bar{\xi}_{1}^{\prime}+\bar{\xi}_{2}^{\prime}\right)}{\bar{\tau} \bar{\xi}_{2}^{\prime}+z \bar{\xi}_{1}^{\prime}}, \xi_{2}=z,
$$

followed by the limit $z \rightarrow-\bar{\tau} \bar{\xi}_{2}^{\prime} / \bar{\xi}_{1}^{\prime}$; and in global pole $\mathcal{G}_{8}$, we should set,

$$
\xi_{2}^{\prime}=\frac{\bar{\xi}_{2}^{\prime}}{z}, \xi_{3}^{\prime}=0, \xi_{3}=-\frac{z+1}{\tau z+1}, \xi_{2}=z,
$$

followed by the limit $z=-1 / \tau$. The solutions and global poles are depicted graphically in fig. 4 . 


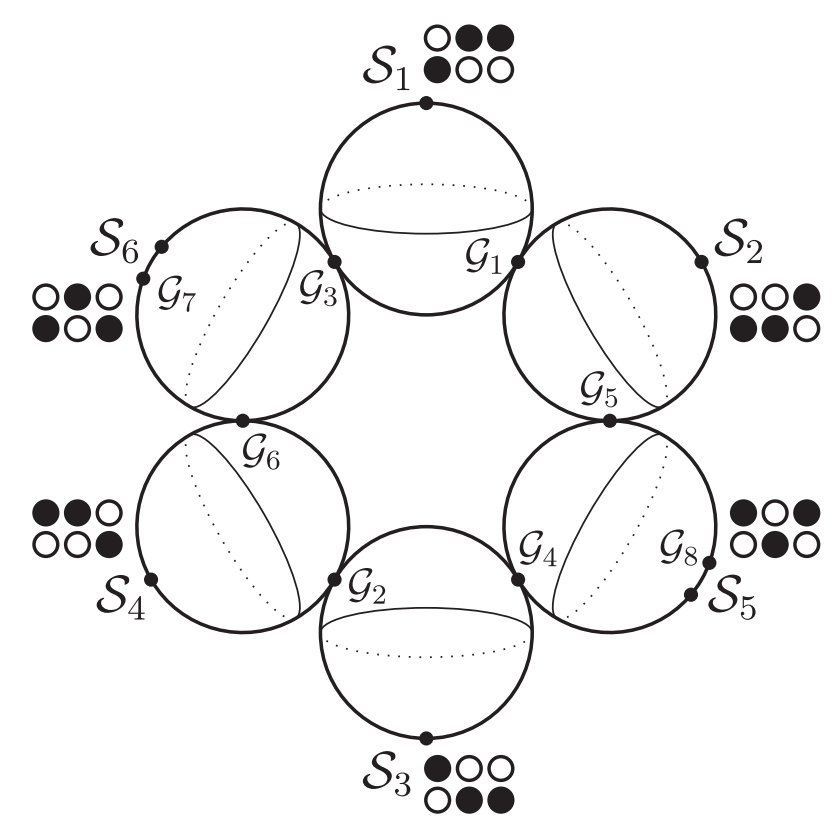

FIG. 5: A representation of the solution space for the class (c) heptacut equations, showing the six independent solutions $\mathcal{S}_{i}$, and the locations of the eight global poles $\mathcal{G}_{j}$. The small white and black blobs indicate the pattern of chiral and antichiral kinematics, respectively, at the vertices of a double-box heptacut.

Within class (c) we find,

$$
J_{\oint}= \begin{cases}J_{\oint}^{(\mathrm{c}, 1)} \equiv-\frac{1}{z(z+1)}, & \text { for solutions } \mathcal{S}_{1,5} \\ J_{\oint}^{(\mathrm{c}, 2)} \equiv \frac{\bar{\xi}_{1}^{\prime}}{z\left(\bar{\xi}_{1}^{\prime}+z\right)}, & \text { for solution } \mathcal{S}_{2} \\ J_{\oint}^{(\mathrm{c}, 3)} \equiv-\frac{\bar{\xi}_{4}^{\prime}}{z\left(\bar{\xi}_{4}^{\prime}+z\right)}, & \text { for solution } \mathcal{S}_{3} \\ J_{\oint}^{(\mathrm{c}, 4)} \equiv \frac{1}{z(z+1)}, & \text { for solution } \mathcal{S}_{4} \\ J_{\oint}^{(\mathrm{c}, 5)} \equiv-\frac{\bar{\xi}_{1}^{\prime}}{z\left(\bar{\xi}_{1}^{\prime}+z\right)}, & \text { for solution } \mathcal{S}_{6}\end{cases}
$$

The first and fourth of these Jacobians have poles at,

$$
\begin{aligned}
& z=0 \\
& z=-1
\end{aligned}
$$


the second and fifth at,

$$
\begin{aligned}
& z=0 \\
& z=-\bar{\xi}_{1}^{\prime}
\end{aligned}
$$

and the third at,

$$
\begin{aligned}
& z=0, \\
& z=-\bar{\xi}_{4}^{\prime} .
\end{aligned}
$$

Here, looking at eq. (3.24), we see that powers of the loop momentum in the numerator will induce poles at $z=\infty$ for all solutions $\mathcal{S}_{i}$; at,

$$
z=-\frac{1}{\tau}
$$

for solution $\mathcal{S}_{5}$; and at,

$$
z=-\frac{\bar{\xi}_{1}^{\prime}}{\bar{\tau}}
$$

for solution $\mathcal{S}_{6}$.

Once again, once the dust settles on comparing ostensibly different solutions, one finds eight independent global poles, with $\xi_{1}^{\prime}=\bar{\xi}_{1}^{\prime}, \xi_{4}^{\prime}=\bar{\xi}_{4}^{\prime}, \zeta_{1}=0=\zeta_{2}$, and the following values of $\left(\xi_{2}, \xi_{2}^{\prime}, \xi_{3}, \xi_{3}^{\prime}\right)$

$$
\begin{array}{ll}
\mathcal{G}_{1}: & \left(0,-\bar{\xi}_{1}^{\prime},-1,0\right), \\
\mathcal{G}_{2}: & \left(-1,0,0,-\bar{\xi}_{4}^{\prime}\right), \\
\mathcal{G}_{3}: & \left(0,-\bar{\xi}_{1}^{\prime}, 0,0\right), \\
\mathcal{G}_{4}: & (-1,0,0,0), \\
\mathcal{G}_{5}: & (0,0,-1,0), \\
\mathcal{G}_{6}: & \left(0,0,0,-\bar{\xi}_{4}^{\prime}\right), \\
\mathcal{G}_{7}: & \left(0,-\frac{\bar{\xi}_{1}^{\prime}}{\bar{\tau}}, 0, \infty\right), \\
\mathcal{G}_{8}: & \left(-\frac{1}{\tau}, 0, \infty, 0\right) .
\end{array}
$$

Once again, for the global poles with an infinite coefficient, we must take the limit carefully; for global pole $\mathcal{G}_{7}$, we should set,

$$
\xi_{2}=0, \xi_{3}=0, \xi_{3}^{\prime}=-\frac{\left(\bar{\xi}_{1}^{\prime}+z\right) \bar{\xi}_{4}^{\prime}}{\bar{\tau} z+\bar{\xi}_{1}^{\prime}}, \xi_{2}^{\prime}=z,
$$


and then take the limit $z \rightarrow-\bar{\xi}_{1}^{\prime} / \bar{\tau}$, while for global pole $\mathcal{G}_{8}$, we should set,

$$
\xi_{2}^{\prime}=0, \xi_{3}^{\prime}=0, \xi_{3}=-\frac{z+1}{\tau z+1}, \xi_{2}=z
$$

and then take the limit $z \rightarrow-1 / \tau$.

The solutions and global poles are depicted graphically in fig. 5.

\section{CONSTRAINT EQUATIONS}

As we have seen in the previous section, each double-box integral with a general numerator has eight independent global poles. One might therefore imagine obtaining eight independent equations for coefficients of integrals by integrating both the left- and right-hand sides of the two-loop version of eq. (1.1) on a $T^{8}$ contour surrounding each global pole in turn,

$$
\oint_{T^{8}\left(\mathcal{G}_{i}\right)} d^{8} v_{a, b} J_{\oint, i} \text { Amplitude Integrand }=\sum_{j \in \text { Basis }} c_{j} \oint_{T^{8}\left(\mathcal{G}_{i}\right)} d^{8} v_{a, b} J_{\oint, i} \text { Integrand }_{j} .
$$

where $T^{8}\left(\mathcal{G}_{i}\right)$ denotes an eight-torus encircling the $i$ th global pole, and where $J_{\oint, i}$ is the corresponding Jacobian derived in the previous section. The $v_{a, b}$, as before, denote the eight variables $\zeta_{1,2}, \xi_{2,3}$, and $\xi_{1,2,3,4}^{\prime}$.

These equations would not all be valid, however, because the derivation of the reduced form on the right-hand side assumes that a number of integrals, with certain kinds of numerators, vanish identically. We must restrict ourselves to contours, whether encircling one global pole or multiple ones, that enforce the vanishing of these integrals. As illustrated in detail in a simple one-loop example in ref. [62], non-vanishing numerators which yield a vanishing integral over the standard real contour will generically not vanish on a linear combination of contours encircling global poles.

Before deriving constraints equations for these contours, we must address a subtlety regarding the orientation of the eight tori $T^{8}\left(\mathcal{G}_{i}\right)$. The global poles $\mathcal{G}_{1}, \ldots, \mathcal{G}_{4}$ in class (b) as well as the global poles $\mathcal{G}_{1}, \ldots, \mathcal{G}_{6}$ in class (c) are shared between two distinct Riemann spheres (see figs. 4 and 5). We adopt the convention that the corresponding residue is to be evaluated on the sphere located towards the anti-clockwise direction of the figures. Thus, in class (b), for example, the residue at $\mathcal{G}_{1}$ should be evaluated from $\mathcal{S}_{1}$; the residue at $\mathcal{G}_{2}$ from $\mathcal{S}_{3}$, etc. Moreover, we choose the orientations on each Riemann sphere such that for 
any global pole $\mathcal{G}_{k} \in \mathcal{S}_{i} \cap \mathcal{S}_{j}$, the residues evaluated from spheres $\mathcal{S}_{i}$ and $\mathcal{S}_{j}$ are equal and opposite. That is, for an arbitrary function $f$ of the loop momenta one has,

$$
\left.\operatorname{Res}_{\mathcal{G}_{k}} J_{\oint} f\left(\ell_{1}, \ell_{2}\right)\right|_{\mathcal{S}_{i}}=-\left.\operatorname{Res}_{\mathcal{G}_{k}} J_{\oint} f\left(\ell_{1}, \ell_{2}\right)\right|_{\mathcal{S}_{j}}
$$

in agreement with the conventions of ref. [71]. Of course, other choices of conventions are possible, but all will lead to the same final results for the two-loop integral coefficients.

Let us now turn to the derivation of constraint equations. We focus on the double-box topology. Denote the insertion of the polynomial $f\left(\ell_{1}, \ell_{2}\right)$ into the numerator of the double box by,

$$
P_{2,2}^{* *}\left[f\left(\ell_{1}, \ell_{2}\right)\right]=\int \frac{d^{D} \ell_{1}}{(2 \pi)^{D}} \frac{d^{D} \ell_{2}}{(2 \pi)^{D}} \frac{f\left(\ell_{1}, \ell_{2}\right)}{\ell_{1}^{2}\left(\ell_{1}-k_{1}\right)^{2}\left(\ell_{1}-K_{12}\right)^{2}\left(\ell_{1}+\ell_{2}\right)^{2} \ell_{2}^{2}\left(\ell_{2}-k_{4}\right)^{2}\left(\ell_{2}-K_{34}\right)^{2}} .
$$

For class (c), there are two master integrals, which we take to be

$$
I_{1}=P_{2,2}^{* *}[1], \quad \text { and } \quad I_{2}=P_{2,2}^{* *}\left[\ell_{1} \cdot k_{4}\right]
$$

For class (b), there are three master integrals, which we take to be,

$$
I_{1}=P_{2,2}^{* *}[1], \quad I_{2}=P_{2,2}^{* *}\left[\ell_{1} \cdot k_{4}\right], \quad \text { and } \quad I_{3}=P_{2,2}^{* *}\left[\ell_{2} \cdot k_{1}\right]
$$

Vanishing integrals with non-vanishing numerators can all be thought of as integrals of total derivatives. They fall into two distinct classes, parity-odd and parity-even. The former consist of five integrals with insertions of Levi-Civita symbols,

$$
\begin{aligned}
& P_{2,2}^{* *}\left[\varepsilon\left(\ell_{1}, k_{2}, k_{3}, k_{4}\right)\right], \quad P_{2,2}^{* *}\left[\varepsilon\left(\ell_{2}, k_{2}, k_{3}, k_{4}\right)\right], \quad P_{2,2}^{* *}\left[\varepsilon\left(\ell_{1}, \ell_{2}, k_{1}, k_{2}\right)\right], \\
& P_{2,2}^{* *}\left[\varepsilon\left(\ell_{1}, \ell_{2}, k_{1}, k_{3}\right)\right], \quad \text { and } \quad P_{2,2}^{* *}\left[\varepsilon\left(\ell_{1}, \ell_{2}, k_{2}, k_{3}\right)\right],
\end{aligned}
$$

whose vanishing on our chosen contours imposes one set of constraints.

The parity-even total derivatives are precisely the integrands which yield the equations required to fully reduce all possible integrals arising in a gauge theory to the two or three master integrals.

Imagine computing a two-loop amplitude with four external momenta diagrammatically. At the first step, the numerator of the two-loop double box will contain dot products of the two loop momenta $\ell_{1,2}$ with external polarization vectors, external momenta, and external spinor strings (if any external legs are fermions). We can express all dot products of loop 
momenta with external vectors in terms of eight dot products: $\ell_{j} \cdot k_{1}, \ell_{j} \cdot k_{2}, \ell_{j} \cdot k_{4}$, and $\ell_{j} \cdot v$, where $v^{\mu}=\varepsilon\left(\mu, k_{1}, k_{2}, k_{4}\right)$. Just as at one loop, odd powers of $v$ will give rise to vanishing integrals, as expressed in the Levi-Civita constraints discussed above. Even powers can again be re-expressed in terms of the other dot products (up to terms involving the $(-2 \epsilon)$ dimensional components of the loop momentum). All integrals can then be rewritten in terms of the six dot products of the loop momenta with the external momenta.

Of these six dot products, three of them $-\ell_{1} \cdot k_{1}, \ell_{1} \cdot k_{2}, \ell_{2} \cdot k_{4}$ - can be rewritten as linear combinations of the propagator denominators and external invariants. One additional dot product of $\ell_{2}$ - say $\ell_{2} \cdot k_{2}$ - can be rewritten in terms of the remaining two $\left(\ell_{1} \cdot k_{4}\right.$ and $\left.\ell_{2} \cdot k_{1}\right)$, propagator denominators, and external invariants. The remaining two dot products are called irreducible.

At first glance, all integrals of the form,

$$
P_{2,2}^{* *}\left[\left(\ell_{2} \cdot k_{1}\right)^{m}\left(\ell_{1} \cdot k_{4}\right)^{n}\right]
$$

would appear to be irreducible. In a general gauge theory, all integrals with $0 \leq m+n \leq 6$ and $m, n \leq 4$ can appear, giving a total of 22 integrals. At a first stage, then, before using integration-by-parts (IBP) identities, we can reduce an arbitrary double-box integral appearing in a gauge-theory amplitude to a linear combination of the 22 different integrals that can arise with powers of the two irreducible numerators. The IBP relations will allow us to reduce the integrals to a small set of master integrals. The number of IBP relations depends on the configuration of external momenta. For class (b), we have 19 such relations, leaving the three masters in eq. (5.5). For class (c), we have 20 such relations, leaving us with the two masters in eq. (5.4). Each IBP relation gives us a nontrivial numerator which integrates to zero; explicit examples may be found in ref. [62].

In order to ensure that these equations continue to hold after performing the global contour integrals, we seek a linear combination of $T^{8}$ contours surrounding the different global poles that satisfies

$$
\sum_{j=1}^{8} a_{j} \oint_{T^{8}\left(\mathcal{G}_{j}\right)} d^{8} v J_{\oint, j} F=0,
$$

and replace $F$ in turn by each of the parity-odd or -even numerators discussed above. This gives us five equations for the parity-odd numerators, and either 19 or 20 for the parity-even ones. 
It turns out that one of the five Levi-Civita equations is linearly dependent on the others, just as in the purely massless case considered in ref. [62]. This leaves us with four independent constraints; in classes (b) and (c), these have the form ${ }^{1}$,

$$
\left(\begin{array}{cccccccc}
1 & -1 & 0 & 0 & 0 & 0 & 0 & 0 \\
0 & 0 & 1 & -1 & 0 & 0 & 0 & 0 \\
0 & 0 & 0 & 0 & 1 & -1 & 0 & 0 \\
0 & 0 & 0 & 0 & 0 & 0 & 1 & -1
\end{array}\right)\left(\begin{array}{l}
a_{1} \\
a_{2} \\
a_{3} \\
a_{4} \\
a_{5} \\
a_{6} \\
a_{7} \\
a_{8}
\end{array}\right)=0
$$

Similarly, only one IBP equation is independent for class (b),

$$
\left(\begin{array}{llllllll}
1 & 1 & -1 & -1 & 1 & 1 & 0 & 0
\end{array}\right)\left(\begin{array}{c}
a_{1} \\
a_{2} \\
a_{3} \\
a_{4} \\
a_{5} \\
a_{6} \\
a_{7} \\
a_{8}
\end{array}\right)=0 ;
$$

and only two for class (c),

$$
\left(\begin{array}{cccccccc}
1 & 1 & -1 & -1 & 0 & 0 & 0 & 0 \\
1 & 1 & 0 & 0 & -1 & -1 & -1 & -1
\end{array}\right)\left(\begin{array}{l}
a_{1} \\
a_{2} \\
a_{3} \\
a_{4} \\
a_{5} \\
a_{6} \\
a_{7} \\
a_{8}
\end{array}\right)=0 .
$$

\footnotetext{
${ }^{1}$ We remark that, in class (b), swapping $\gamma_{12}^{+} \longleftrightarrow \gamma_{12}^{-}$amounts to changing the coordinate value at the location of the global poles as well as changing the value of the corresponding residue. The constraint equations (5.9) and (5.10) are invariant.
} 
The simultaneous solutions to these equations are choices of contours suitable for integrating on both sides of the two-loop version of eq. (1.1) in order to derive useful relations for coefficients.

\section{PROJECTORS}

Imposing the constraints obtained in the previous section will leave us with three nontrivial linearly independent combinations of contours for external kinematics in class (b), and two combinations in class (c). Using these contours on the two-loop version of eq. (1.1) restricted to four-point amplitudes will then give us three or two equations, respectively, for the coefficients of the three or two different master integrals (5.5) and (5.4). We can diagonalize these equations by requiring in turn the vanishing of each master integral along the contour. The resulting equations for the coefficients take the form,

$$
c_{i}=\sum_{j=1}^{8} a_{j} \oint_{T^{8}\left(\mathcal{G}_{j}\right)} d^{8} v_{a, b} J_{\oint, j} \sum_{\substack{\text { particles } \\ \text { helicities }}} \prod_{p=1}^{6} A_{p}^{(0)}\left(v_{a, b}\right),
$$

where the product in the integrand is over the tree amplitudes at all vertices, with their dependence coming through the parametrization (2.7)-(2.8). The product is then summed over all allowed choices of internal particles and helicities.

This abstract form of a multidimensional contour integration hides a subtlety whose full exploration we postpone to a future paper. In multivariate complex contour integration, the result is not necessarily independent of the order of integration. In our context, this is connected with accidental sharing of singularities between different integrals, a feature not present in the one-loop case. Once we specify the ordering, the accidental sharing disappears, as does any ambiguity in the result. In the present context, the order in eq. (6.1) is specified by performing the $z$ integration last,

$$
\left.\oint_{T^{8}\left(\mathcal{G}_{j}\right)} d^{8} v J_{\oint} \mathcal{I} \equiv \oint_{C_{\delta}\left(z_{j}^{0}\right)} d z J_{\oint, j} \mathcal{I}\right|_{\mathcal{S}\left(\mathcal{G}_{j}\right)}
$$

where the integrand is first to be evaluated at a heptacut solution $\mathcal{S}\left(\mathcal{G}_{j}\right)$ containing the global pole, and the contour integral in $z$ is then to be performed on a small circle $C_{\delta}$ surrounding the value of $z$ at the given global pole. In this equation as well as in eq. (6.1), $J_{\oint, j}$ refers to the Jacobian of the chosen heptacut solution. (As remarked at the beginning of Section $\mathrm{V}$, 
there is more than one heptacut solution containing a given global pole; we refer to the discussion there for our conventions.)

In class (b), the residues for the three master integrals are given by

$$
\begin{array}{ll}
I_{1}: & R_{1}=(1,1,1,1,0,0,0,0) \\
I_{2}: & R_{2}=\frac{\gamma_{12}\left(s_{12} s_{14}-m_{1}^{2} m_{3}^{2}\right)}{2\left(\gamma_{12}^{2}-m_{1}^{2} m_{2}^{2}\right)}(0,0,0,0,1,1,0,0) \\
I_{3}: & R_{3}=\frac{s_{12}\left(s_{14}-m_{1}^{2}\right)}{2 \gamma_{34}}(1,1,1,1,0,0,0,0)+\frac{m_{1}^{2} m_{3}^{2}-s_{12} s_{14}}{2 \gamma_{34}}(1,1,0,0,0,0,1,1) .
\end{array}
$$

Imposing the constraints (5.9) and (5.10) and then inverting the matrix of these residue vectors, we obtain projectors (or master contours) isolating a single master integral. Each is specified by a vector giving the values of the $a_{i}$ to be used in the integrations above. In class (b), the projectors for the three masters are,

$$
\begin{aligned}
& I_{1}: \quad P_{1}=\frac{1}{4}\left(1,1,1,1,0,0,1-\frac{2 m_{1}^{2}\left(m_{3}^{2}-s_{12}\right)}{m_{1}^{2} m_{3}^{2}-s_{12} s_{14}}, 1-\frac{2 m_{1}^{2}\left(m_{3}^{2}-s_{12}\right)}{m_{1}^{2} m_{3}^{2}-s_{12} s_{14}}\right), \\
& I_{2}: \quad P_{2}=\frac{N_{2}^{(\mathrm{b})}}{2}(-1,-1,1,1,2,2,1,1), \\
& I_{3}: \quad P_{3}=\frac{N_{3}^{(\mathrm{b})}}{2}(0,0,0,0,0,0,-1,-1),
\end{aligned}
$$

where $^{2}$,

$$
\begin{aligned}
& N_{2}^{(\mathrm{b})}=\frac{\gamma_{12}^{2}-m_{1}^{2} m_{2}^{2}}{\gamma_{12}\left(s_{12} s_{14}-m_{1}^{2} m_{3}^{2}\right)}, \\
& N_{3}^{(\mathrm{b})}=\frac{2\left(s_{12}-m_{3}^{2}\right)}{s_{12} s_{14}-m_{1}^{2} m_{3}^{2}} .
\end{aligned}
$$

The formulæ in previous sections allow for any pattern of masses within class (b); but the residues in eq. (6.3) and the projectors here are given for the special case that $m_{4}=0$, $m_{1} \neq 0 \neq m_{2}$, and hold whether $m_{3}$ is zero or not.

In class (c), the residues for the two master integrals are given by

$$
\begin{array}{ll}
I_{1}: & R_{1}=(1,1,1,1,1,1,0,0) \\
I_{2}: & R_{2}=-\frac{1}{2}\left(m_{4}^{2}, m_{4}^{2}, m_{4}^{2}, m_{4}^{2},-2 k_{1}^{b} \cdot k_{4}\left(1+\frac{m_{2}^{2}}{\gamma_{12}}\right),-2 k_{1}^{b} \cdot k_{4}\left(1+\frac{m_{2}^{2}}{\gamma_{12}}\right), 0,0\right) .
\end{array}
$$

\footnotetext{
${ }^{2}$ For the normalization factor $N_{2}^{(\mathrm{b})}$ in the projector for integral $I_{2}$, swapping $\gamma_{12}^{+} \longleftrightarrow \gamma_{12}^{-}$will induce a change of sign in the value of $N_{2}^{(\mathrm{b})}$ and the heptacut of $I_{2}$.
} 
In class (c), the projectors are

$$
\begin{aligned}
I_{1}: \quad P_{1}= & \frac{N_{1}^{(c)}}{4}\left(1,1,1,1, \frac{2 m_{4}^{2}\left(s_{12}-m_{1}^{2}\right)}{s_{12}\left(s_{14}-m_{4}^{2}\right)+m_{1}^{2} m_{4}^{2}}, \frac{2 m_{4}^{2}\left(s_{12}-m_{1}^{2}\right)}{s_{12}\left(s_{14}-m_{4}^{2}\right)+m_{1}^{2} m_{4}^{2}},\right. \\
1 & \left.-\frac{2 m_{4}^{2}\left(s_{12}-m_{1}^{2}\right)}{s_{12}\left(s_{14}-m_{4}^{2}\right)+m_{1}^{2} m_{4}^{2}}, 1-\frac{2 m_{4}^{2}\left(s_{12}-m_{1}^{2}\right)}{s_{12}\left(s_{14}-m_{4}^{2}\right)+m_{1}^{2} m_{4}^{2}}\right), \\
I_{2}: \quad P_{2}= & \frac{N_{2}^{(c)}}{4}(1,1,1,1,-2,-2,3,3),
\end{aligned}
$$

where

$$
\begin{aligned}
& N_{1}^{(\mathrm{c})}=1+\frac{m_{4}^{2}\left(m_{1}^{2}-s_{12}\right)}{s_{12} s_{14}}, \\
& N_{2}^{(\mathrm{c})}=-\frac{1}{k_{1}^{b} \cdot k_{4}}\left(1+\frac{m_{4}^{2}\left(m_{1}^{2}-s_{12}\right)}{s_{12} s_{14}}\right) .
\end{aligned}
$$

As above, the formulæ in previous sections and in eq. (6.6) allow for any pattern of masses within class (c); but the projectors here are given for the special case that $m_{2}=0, m_{1} \neq 0$, and at most one of $m_{3}$ and $m_{4}$ is non-zero.

\section{EXAMPLES: HIGGS AMPLITUDES AND FORM FACTORS}

In this section we will work out cut expressions, residues and integral coefficients for the one-mass double-box integral in a few simple but nontrivial four-point examples. We use these examples to illustrate how to apply the method described in previous sections. In particular we discuss a single massive scalar amplitude and a form factor. A simple way to introduce a massive scalar in a gauge invariant way is to add the following interaction to the pure Yang-Mills theory:

$$
\mathcal{L}_{\text {int. }}=-\frac{1}{4 \Lambda} H \operatorname{Tr}\left(F^{\mu \nu} F_{\mu \nu}\right),
$$

where $\Lambda$ denotes the scale at which the physics underlying this higher-dimension operator has been integrated out, and $H$ is a massive scalar boson. This is the simplest gluon-scalar interaction for a colorless scalar not carrying a conserved charge. It is precisely the form of the effective Lagrangian [82] for the Standard Model Higgs-gluon interaction, once the

top quark has been integrated out. Recently, Gehrmann et al. have computed the two-loop $H \rightarrow$ ggg amplitude in this effective theory [83], later simplified by Duhr [84]. 
The computation of the scalar's three-gluon decay amplitude can also be viewed as the computation of the three-gluon form factor for the operator,

$$
\mathcal{O}_{g} \equiv-\frac{1}{4} \operatorname{Tr}\left(F^{\mu \nu} F_{\mu \nu}\right) .
$$

We can compute such form factors using the same approach as for on-shell amplitudes,

$$
\langle 0|\mathcal{O}(q)| 1,2, \ldots, n\rangle=A^{\mathcal{O}}(q, 1,2, \ldots, n) .
$$

In addition to the operator $\mathcal{O}_{g}$, we will also consider a well-studied scalar operator in $\mathcal{N}=4$ super-Yang-Mills theory (SYM),

$$
\mathcal{O}_{s} \equiv \frac{1}{2} \operatorname{Tr}\left(\phi_{12} \phi_{12}\right)
$$

where the subscripts refer to $R$-charge indices. These indices will not play a role here; one can think of $\phi_{12}$ as simply a complex scalar with a conserved $U(1)$ charge: $\phi^{12} \equiv \phi^{+}=\phi$ and $\phi_{12} \equiv \phi^{-}=\bar{\phi}$. Brandhuber, Travaglini and Yang recently computed the three-particle form factor of this operator at two loops [85]. We compare our calculation of the double-box coefficients to their results.

For our computations, the only tree-level ingredients we need are the three-point amplitudes and two-particle form factors. The nonvanishing form factors, after stripping off color factors, are,

$$
\left\langle 1_{g}^{+}, 2_{g}^{+}\left|\mathcal{O}_{g}\right| 0\right\rangle=[12]^{2}, \quad\left\langle 1_{g}^{-}, 2_{g}^{-}\left|\mathcal{O}_{g}\right| 0\right\rangle=\langle 12\rangle^{2}, \quad\left\langle 1_{s}^{+}, 2_{s}^{+}\left|\mathcal{O}_{s}\right| 0\right\rangle=1
$$

(We omit an overall dimensionful factor of $1 / \Lambda$.)

\section{A. Computing the integral coefficients}

Let us follow the procedure derived in previous sections. First we form the product of amplitudes appearing in eq. (6.1), evaluated at each of the heptacut solutions $\mathcal{S}_{1}, \ldots, \mathcal{S}_{6}$, depicted in fig. 6. This is the integrand of our remaining contour integral. This corresponds to evaluating the factor $\mathcal{I}$ in eq. (6.2) at each of the solutions $\mathcal{S}_{i}$.

Next, we evaluate the remaining contour integral in $z$ by residues. Here, we should distinguish between two types of poles: those arising from the heptacut Jacobian (4.22)(4.24); and those arising from powers of the loop momentum in the integrand itself (4.25)(4.26). The integrand can safely be evaluated at the former type, where it is finite. 

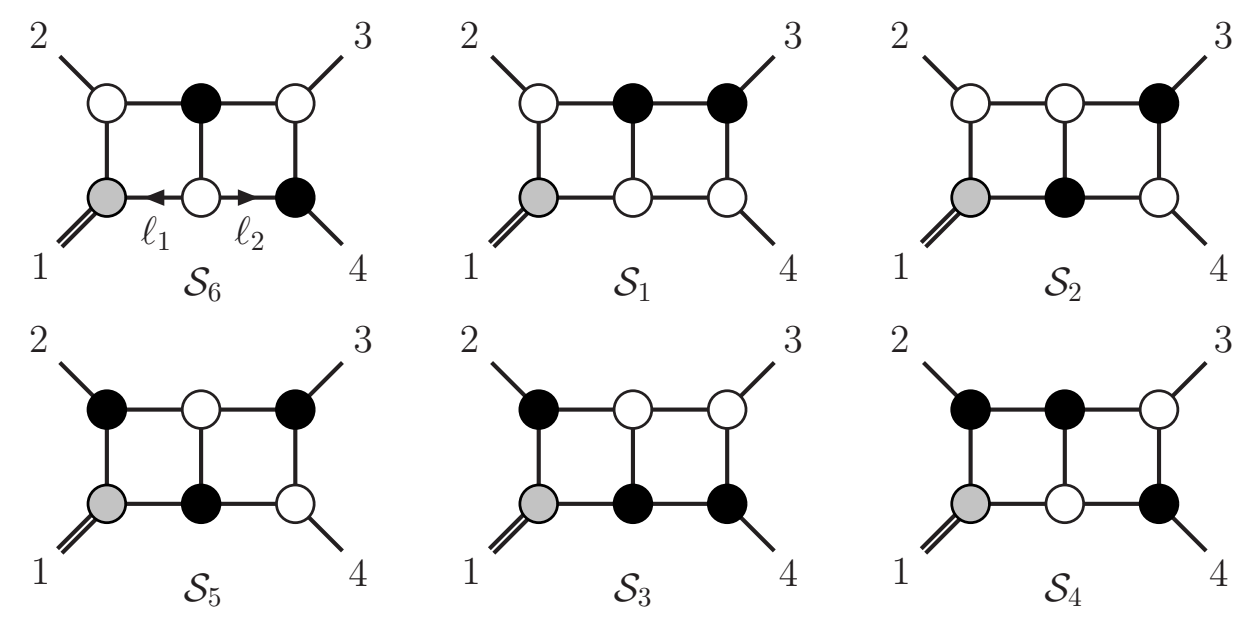

FIG. 6: The six solutions to the heptacut kinematics of a one-mass double box. White and black blobs indicate chiral and antichiral kinematics at the vertices, respectively. The gray blobs denote vertices with no definite chirality.

The poles arising from powers of the loop momentum are at infinite values of one of the loop momenta. (The poles are nonetheless at finite values of the remaining integration variable z.) The Jacobian factor remains finite at these poles. Here the procedure is more involved, because in general the poles may be of higher order. The order of the pole depends on the ultraviolet power-counting behavior of the process in the given theory. In $\mathcal{N}=4$ SYM, and for the form factors we consider here, the poles will be simple. A general fermion and scalar content in the theory would lead to poles of higher order. (From a computational point of view, the residues at infinite loop momentum require a more careful treatment as they may receive contributions that arise from the interference between singular and finite terms in the Laurent expansion of the various loop-momentum factors entering the integrand.)

For each kinematical solution $\mathcal{S}_{j}$ we denote the integrand by $\mathcal{I}_{j}$,

$$
\left.\mathcal{I}_{j} \equiv(-i)^{7} \sum_{\substack{\text { particlese } \\ \text { helicities }}} \prod_{i=1}^{6} A_{i}^{(0)}\left(\ell_{1}, \ell_{2}\right)\right|_{\mathcal{S}_{j}} .
$$

At each global pole, the remaining integrand in $z$ has a pole at a single location $z_{j}^{0}$; we evaluate the residue at that pole,

$$
R_{\mathcal{G}_{j}}=\operatorname{Res}_{z=z_{j}^{0}} J_{\oint, j} \mathcal{I}\left(\mathcal{G}_{j}\right)
$$



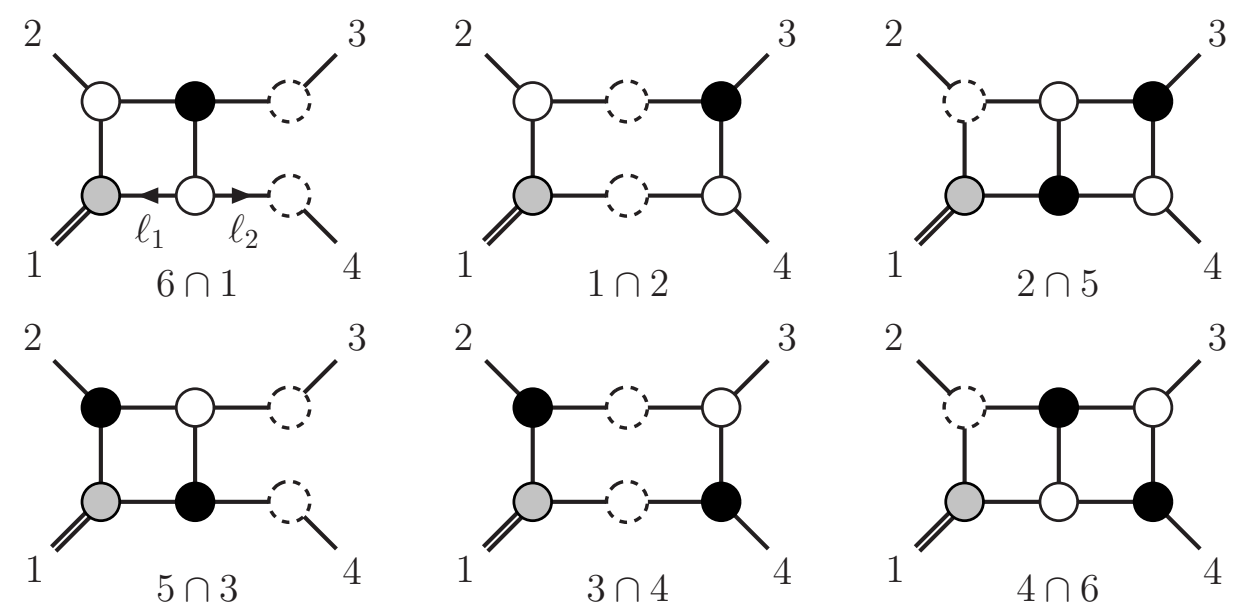

FIG. 7: The Jacobian poles are located at the intersection points of the six kinematical solutions, denoted by $i \cap j$ for solutions $\mathcal{S}_{i}$ and $\mathcal{S}_{j}$. They correspond to situations where vertical rungs become soft (in the massless case), as indicated by the absent lines, or in a more general situation where the kinematics becomes collinear, as indicated by the dashed blobs.

as described above.

The results below are valid for any massless gauge theory with $n_{f}$ fermions and $n_{s}$ (complex) scalars, both in the adjoint representation. The kinematical solutions and poles in the one-mass case are illustrated in fig. 6] and fig. 7, respectively. We can read off the amplitudes from the pattern of white and black blobs in the figures. Maximally helicity violating (MHV) amplitudes are non-vanishing for chiral kinematics, but vanish for antichiral kinematics. The white blobs therefore give rise to MHV amplitudes. Similarly, $\overline{\mathrm{MHV}}$ amplitudes are non-vanishing for antichiral kinematics, but vanish for chiral kinematics. The black blobs therefore give rise to $\overline{\mathrm{MHV}}$ amplitudes. More detailed information on notation

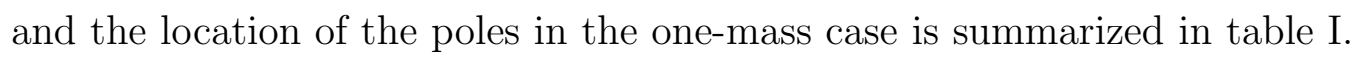

\section{The $\left(\mathcal{O}_{g}, 2^{-}, 3^{-}, 4^{-}\right)$Configuration}

It will be helpful to label the internal cut lines as follows,

$$
\ell_{3}=\ell_{1}-k_{1}, \ell_{4}=\ell_{1}-k_{1}-k_{2}, \ell_{5}=\ell_{2}-k_{4}, \ell_{6}=\ell_{2}-k_{4}-k_{3}, \ell_{7}=-\ell_{1}-\ell_{2} .
$$

For each heptacut solution, compute the cut integrand by multiplying together tree amplitudes for each vertex, and summing over different possible assignments of internal states. 
TABLE I: The properties of the global poles in the one-mass case: Here we assume that $m_{2}=$ $m_{3}=m_{4}=0$. The notation $i \cap j$ indicates that the pole is located at the intersection point of the Riemann spheres labeled by $\mathcal{S}_{i}$ and $\mathcal{S}_{j}$, and $\infty_{i R}$ corresponds to poles in $\mathcal{S}_{i}$ at infinite $\ell_{2}$ momentum (see ref. [71]).

\begin{tabular}{|c|c|c|c|c|}
\hline pole & location & $\left(\xi_{2}, \xi_{2}^{\prime}, \xi_{3}, \xi_{3}^{\prime}\right)$ & $\ell_{1}$ & $\ell_{2}$ \\
\hline $\mathcal{G}_{1}$ & $1 \cap 2$ & $(0,-1,-1,0)$ & $-\frac{1}{2}\left[4\left|\gamma^{\mu}\right| 1^{b}\right\rangle \frac{\langle 43\rangle}{\left\langle 1^{b} 3\right\rangle}$ & $\frac{1}{2}\left[4\left|\gamma^{\mu}\right| 1^{b}\right\rangle \frac{\langle 43\rangle}{\left\langle 1^{b} 3\right\rangle}$ \\
\hline $\mathcal{G}_{2}$ & $3 \cap 4$ & $(-1,0,0,-1)$ & $-\frac{1}{2}\left\langle 4\left|\gamma^{\mu}\right| 1^{b}\right] \frac{[43]}{\left[1^{D} 3\right]}$ & $\frac{1}{2}\left\langle 4\left|\gamma^{\mu}\right| 1^{b}\right] \frac{[43]}{\left[1^{D} 3\right]}$ \\
\hline $\mathcal{G}_{3}$ & $6 \cap 1$ & $(0,-1,0,0)$ & $-\frac{1}{2}\left[4\left|\gamma^{\mu}\right| 1^{b}\right\rangle \frac{\langle 43\rangle}{\left\langle 1^{b} 3\right\rangle}$ & $k_{4}$ \\
\hline $\mathcal{G}_{4}$ & $5 \cap 3$ & $(-1,0,0,0)$ & $-\frac{1}{2}\left\langle 4\left|\gamma^{\mu}\right| 1^{b}\right] \frac{[43]}{\left[1^{\mathrm{p}} 3\right]}$ & $k_{4}$ \\
\hline $\mathcal{G}_{5}$ & $2 \cap 5$ & $(0,0,-1,0)$ & $k_{1}^{b}$ & $\frac{1}{2}\left[4\left|\gamma^{\mu}\right| 1^{b}\right\rangle \frac{\langle 43\rangle}{\left\langle 1^{\supset} 3\right\rangle}$ \\
\hline $\mathcal{G}_{6}$ & $4 \cap 6$ & $(0,0,0,-1)$ & $k_{1}^{b}$ & $\frac{1}{2}\left\langle 4\left|\gamma^{\mu}\right| 1^{b}\right] \frac{[43]}{\left[1^{b} 3\right]}$ \\
\hline $\mathcal{G}_{7}$ & $\infty_{6 R}$ & $\left(0,-\frac{\left[31^{b}\right][42]}{[32]\left[41^{D}\right]}, 0, \infty\right)$ & $-\frac{1}{2}\left[3\left|\gamma^{\mu}\right| 1^{b}\right\rangle \frac{\langle 34\rangle}{\left\langle 1^{D} 4\right\rangle}$ & $\infty\left[3\left|\gamma^{\mu}\right| 4\right\rangle$ \\
\hline $\mathcal{G}_{8}$ & $\infty_{5 R}$ & $\left(-\frac{\left\langle 31^{b}\right\rangle\langle 42\rangle}{\langle 32\rangle\left\langle 41^{D}\right\rangle}, 0, \infty, 0\right)$ & $-\frac{1}{2}\left\langle 3\left|\gamma^{\mu}\right| 1^{b}\right] \frac{[34]}{\left[1^{p} 4\right]}$ & $\infty\left\langle 3\left|\gamma^{\mu}\right| 4\right]$ \\
\hline
\end{tabular}

For example, for the evaluation on $\mathcal{S}_{6}$, here only a single configuration of internal helicity assignments contributes,

$$
\begin{aligned}
\mathcal{I}_{6}= & (-i)^{7} A^{\mathrm{Higgs}}\left(1^{\mathrm{H}}, \ell_{3}^{-},-\ell_{1}^{-}\right) A^{(0)}\left(2^{-}, \ell_{4}^{-},-\ell_{3}^{+}\right) A^{(0)}\left(3^{-},-\ell_{5}^{-}, \ell_{6}^{+}\right) A^{(0)}\left(4^{-},-\ell_{2}^{+}, \ell_{5}^{+}\right) \\
& \times A^{(0)}\left(\ell_{1}^{+}, \ell_{7}^{-}, \ell_{2}^{-}\right) A^{(0)}\left(-\ell_{4}^{+},-\ell_{6}^{-},-\ell_{7}^{+}\right) \\
= & -\left\langle\ell_{1} \ell_{3}\right\rangle^{2} \frac{\left\langle 2 \ell_{4}\right\rangle^{3}}{\left\langle\ell_{4} \ell_{3}\right\rangle\left\langle\ell_{3} 2\right\rangle} \frac{\left\langle 3 \ell_{5}\right\rangle^{3}}{\left\langle\ell_{5} \ell_{6}\right\rangle\left\langle\ell_{6} 3\right\rangle} \frac{\left[\ell_{2} \ell_{5}\right]^{3}}{\left[\ell_{5} 4\right]\left[4 \ell_{2}\right]} \frac{\left\langle\ell_{7} \ell_{2}\right\rangle^{3}}{\left\langle\ell_{2} \ell_{1}\right\rangle\left\langle\ell_{1} \ell_{7}\right\rangle} \frac{\left[\ell_{7} \ell_{4}\right]^{3}}{\left[\ell_{4} \ell_{6}\right]\left[\ell_{6} \ell_{7}\right]} \\
= & m_{1}^{4} \frac{\left\langle 2\left|1 \ell_{1}\right| 4\right\rangle\langle 43\rangle}{[23]}
\end{aligned}
$$

where the last line is obtained after using some spinor manipulations.

The integrand takes the same simple form on $\mathcal{S}_{1}$ and $\mathcal{S}_{2}$, whereas it vanishes identically on $\mathcal{S}_{5}, \mathcal{S}_{3}$ and $\mathcal{S}_{4}$, that is

$$
\mathcal{I}_{6}=\mathcal{I}_{1}=\mathcal{I}_{2}=m_{1}^{4} \frac{\left\langle 2\left|1 \ell_{1}\right| 4\right\rangle\langle 43\rangle}{[23]}, \quad \mathcal{I}_{5}=\mathcal{I}_{3}=\mathcal{I}_{4}=0 .
$$

These results are the same independent of the number of scalars or fermions in the gauge theory. In particular this means that, for the $\left(\mathcal{O}_{g}, 2^{-}, 3^{-}, 4^{-}\right)$configuration, all (massless) gauge theories have the same double-box cuts as the $\mathcal{N}=4$ theory, explaining the simple dependence on loop momenta in eq. (7.10). 
To obtain the eight residues we should evaluate the integrand in the neighborhood of the global poles, obtaining

$$
\begin{aligned}
\mathcal{I}_{6,1}\left(\mathcal{G}_{3}\right) & =\mathcal{I}_{1,2}\left(\mathcal{G}_{1}\right)=m_{1}^{4} s_{34} \frac{\langle 23\rangle\langle 34\rangle}{[24]}, \\
\mathcal{I}_{6}\left(\mathcal{G}_{7}\right) & =m_{1}^{4} s_{34} \frac{\langle 24\rangle\langle 43\rangle}{[23]} \\
\mathcal{I}_{5}\left(\mathcal{G}_{8}\right) & =\mathcal{I}_{2,5}\left(\mathcal{G}_{5}\right)=\mathcal{I}_{5,3}\left(\mathcal{G}_{4}\right)=\mathcal{I}_{3,4}\left(\mathcal{G}_{2}\right)=\mathcal{I}_{4,6}\left(\mathcal{G}_{6}\right)=0
\end{aligned}
$$

where the notation $\mathcal{I}_{i, j}$ indicates that either solution $\mathcal{S}_{i}$ or solution $\mathcal{S}_{j}$ can be used. The integrand happens to be finite at all global poles, so only the Jacobian poles can give rise to nonvanishing residues. As mentioned, the Jacobian has only simple poles at the intersection points of the kinematical solutions. With our normalization, there is no additional dimensionful quantity that appears (it was removed in the transition from $J_{\oint}^{(0)}$ to $J_{\oint}$ ).

Finally, after multiplying the integrand points $\mathcal{I}_{i}$ by the Jacobian factors, there are exactly two nonzero residues:

$$
R_{\mathcal{G}_{3}}=R_{\mathcal{G}_{1}}=m_{1}^{4} s_{34} \frac{\langle 23\rangle\langle 34\rangle}{[24]} .
$$

Note that $R_{\mathcal{G}_{7}}=0$ even though the integrand is nonvanishing at this point. In vector notation the residues are

$$
R=m_{1}^{4} s_{34} \frac{\langle 23\rangle\langle 34\rangle}{[24]}(1,0,1,0,0,0,0,0)
$$

The integral coefficients are then given by a sum over appropriately weighted residues. For the double box $P_{2,2}^{* *}[1]$ we have

$$
c_{1}=P_{1} \cdot R=\frac{1}{4}\left(R_{\mathcal{G}_{1}}+R_{\mathcal{G}_{2}}+R_{\mathcal{G}_{3}}+R_{\mathcal{G}_{4}}+R_{\mathcal{G}_{7}}+R_{\mathcal{G}_{8}}\right)=\frac{1}{2} m_{1}^{4} s_{34} \frac{\langle 23\rangle\langle 34\rangle}{[24]},
$$

and for the double box $P_{2,2}^{* *}\left[\ell_{1} \cdot k_{4}\right]$,

$$
\begin{aligned}
c_{2}=P_{2} \cdot R & =-\frac{1}{4 k_{1}^{b} \cdot k_{4}}\left(R_{\mathcal{G}_{1}}+R_{\mathcal{G}_{2}}+R_{\mathcal{G}_{3}}+R_{\mathcal{G}_{4}}-2 R_{\mathcal{G}_{5}}-2 R_{\mathcal{G}_{6}}+3 R_{\mathcal{G}_{7}}+3 R_{\mathcal{G}_{8}}\right) \\
& =-\frac{1}{2} m_{1}^{4} s_{34} \frac{\langle 23\rangle\langle 34\rangle}{[24]} \frac{1}{k_{1}^{b} \cdot k_{4}}
\end{aligned}
$$

where $P_{1}$ and $P_{2}$ are given in eq. (6.7). 


\section{The $\left(\mathcal{O}_{g}, 2^{+}, 3^{-}, 4^{-}\right)$Configuration}

For this configuration, we simply quote the main results. The integrand evaluated on the solutions yields,

$$
\mathcal{I}_{6}=\mathcal{I}_{1}=\mathcal{I}_{2}=0, \quad \mathcal{I}_{5}=\mathcal{I}_{3}=\mathcal{I}_{4}=s_{34} \frac{\langle 34\rangle^{3}\left[4\left|\ell_{1} 1\right| 2\right]}{\langle 32\rangle}
$$

valid for any number of fermions and scalars.

Because the integrands are at most linear in momenta the poles will be simple. It is then straightforward to evaluate the residues in each case by plugging in the leading momentum behavior at the pole. The only nonvanishing residues are

$$
R_{\mathcal{G}_{4}}=R_{\mathcal{G}_{2}}=s_{34}^{2} \frac{\langle 34\rangle^{3}[32]}{\langle 24\rangle}
$$

or alternatively

$$
R=s_{34}^{2} \frac{\langle 34\rangle^{3}[32]}{\langle 24\rangle}(0,1,0,1,0,0,0,0) .
$$

The integral coefficients are then given by

$$
c_{1}=P_{1} \cdot R=\frac{1}{2} s_{34}^{2} \frac{\langle 34\rangle^{3}[32]}{\langle 24\rangle},
$$

and

$$
c_{2}=P_{2} \cdot R=-\frac{1}{2} s_{34}^{2} \frac{\langle 34\rangle^{3}[32]}{\langle 24\rangle} \frac{1}{k_{1}^{b} \cdot k_{4}} .
$$

3. The $\left(\mathcal{O}_{g}, 2^{-}, 3^{+}, 4^{-}\right)$Configuration

For this configuration, the results for the various solutions are,

$$
\begin{aligned}
\mathcal{I}_{6}= & \mathcal{I}_{1}=\mathcal{I}_{2}=0, \quad \mathcal{I}_{3}=\mathcal{I}_{4}=\left[2\left|1 \ell_{1}\right| 3\right][34]\langle 42\rangle^{3} \\
\mathcal{I}_{5}= & {\left[2\left|1 \ell_{1}\right| 3\right][34]\langle 42\rangle^{3}+\left(n_{f}-4\right) \frac{\left\langle 2\left|\ell_{1}\right| 3\right]\left\langle 3\left|\ell_{2}\right| 4\right\rangle\langle 42\rangle\left\langle 2\left|1 \ell_{1} \ell_{2}\right| 2\right\rangle}{s_{34}} } \\
& +2\left(n_{s}-n_{f}+1\right) \frac{\left\langle 3\left|\ell_{2}\right| 4\right\rangle^{2}\left\langle 2\left|\ell_{2} \ell_{1}\right| 2\right\rangle\left[2\left|1 \ell_{1} \ell_{2}\right| 2\right\rangle}{s_{34}^{2}}
\end{aligned}
$$

where we have organized the $\mathcal{I}_{5}$ expression such that first term is the $\mathcal{N}=4$ SYM contribution, the second term is the contribution from a chiral $\mathcal{N}=1$ multiplet, and the last term is the scalar contribution. Note that the fermions and scalars only propagate in the rightmost loop, so any potential Yukawa couplings are not relevant here. 
All integrands but $\mathcal{I}_{5}$ are linear in momenta, giving simple poles. The $\mathcal{I}_{5}$ expression has up to fourth order poles. Somewhat surprisingly, upon careful analysis, the residues at the simple poles receive no contribution from the fermions and scalars. All nonvanishing residues are again equal,

$$
R_{\mathcal{G}_{4}}=R_{\mathcal{G}_{2}}=s_{34}[23]\langle 42\rangle^{3}[34]
$$

or alternatively

$$
R=s_{34}[23]\langle 42\rangle^{3}[34](0,1,0,1,0,0,0,0) .
$$

The integral coefficients are

$$
c_{1}=P_{1} \cdot R=\frac{1}{2} s_{34}[23]\langle 42\rangle^{3}[34]
$$

and

$$
c_{2}=P_{2} \cdot R=-\frac{1}{2} s_{34}[23]\langle 42\rangle^{3}[34] \frac{1}{k_{1}^{b} \cdot k_{4}} .
$$

\section{The $\left(\mathcal{O}_{g}, 2^{-}, 3^{-}, 4^{+}\right)$Configuration}

For this configuration the integrands are,

$$
\begin{aligned}
\mathcal{I}_{6}= & \mathcal{I}_{1}=\mathcal{I}_{2}=0, \quad \mathcal{I}_{5}=\mathcal{I}_{4}=\left[2\left|1 \ell_{1}\right| 4\right][43]\langle 32\rangle^{3} \\
\mathcal{I}_{3}= & {\left[2\left|1 \ell_{1}\right| 4\right][43]\langle 32\rangle^{3}+\left(n_{f}-4\right) \frac{\left\langle 2\left|\ell_{1}\right| 4\right]\left[4\left|\ell_{2}\right| 3\right\rangle\langle 32\rangle\left[2\left|1 \ell_{1} \ell_{2}\right| 2\right\rangle}{s_{34}} } \\
& +2\left(n_{s}-n_{f}+1\right) \frac{\left[4\left|\ell_{2}\right| 3\right\rangle^{2}\left\langle 2\left|\ell_{2} \ell_{1}\right| 2\right\rangle\left[2\left|1 \ell_{1} \ell_{2}\right| 2\right\rangle}{s_{34}^{2}}
\end{aligned}
$$

A helpful observation is that these integrands may be related directly to integrand results for the $\left(\mathcal{O}_{g}, 2^{-}, 3^{+}, 4^{-}\right)$configuration, by swapping $k_{3} \leftrightarrow k_{4}, \mathcal{I}_{3} \leftrightarrow \mathcal{I}_{5}$ (and $\mathcal{I}_{1} \leftrightarrow \mathcal{I}_{6}$ ). This is a quite general feature as it follows from using the BCJ [86] amplitude relation $\left(\ell_{2}-k_{4}\right)^{2} A^{(0)}\left(3,4,-\ell_{2}, \ell_{6}\right)=\left(\ell_{2}-k_{3}\right)^{2} A^{(0)}\left(4,3,-\ell_{2}, \ell_{6}\right)$ for the product of trees that $k_{3}$ and $k_{4}$ attach to.

As before the residues have no contribution from the fermions and scalars, and the nonvanishing residues are equal,

$$
R_{\mathcal{G}_{4}}=R_{\mathcal{G}_{2}}=\frac{s_{23} s_{34}\langle 23\rangle^{3}[34]}{\langle 24\rangle},
$$

or alternatively,

$$
R=\frac{s_{23} s_{34}\langle 23\rangle^{3}[34]}{\langle 24\rangle}(0,1,0,1,0,0,0,0) .
$$


The integral coefficients are,

$$
c_{1}=P_{1} \cdot R=\frac{1}{2} \frac{s_{23} s_{34}\langle 23\rangle^{3}[34]}{\langle 24\rangle},
$$

and

$$
c_{2}=P_{2} \cdot R=-\frac{1}{2} \frac{s_{23} s_{34}\langle 23\rangle^{3}[34]}{\langle 24\rangle} \frac{1}{k_{1}^{b} \cdot k_{4}} .
$$

Finally, we note that the remaining nonvanishing helicity configurations $\left(\mathcal{O}_{g}, 2^{+}, 3^{+}, 4^{+}\right)$, $\left(\mathcal{O}_{g}, 2^{-}, 3^{+}, 4^{+}\right),\left(\mathcal{O}_{g}, 2^{+}, 3^{-}, 4^{+}\right)$and $\left(\mathcal{O}_{g}, 2^{+}, 3^{+}, 4^{-}\right)$can be obtained by conjugating spinors in the results quoted above: that is, using $\langle i j\rangle \leftrightarrow[j i]$.

\section{B. Scalar operator}

The scalar operator $\mathcal{O}_{s}=\frac{1}{2} \operatorname{Tr}\left(\phi_{12} \phi_{12}\right)$ carries $R$-charge. To obtain a non-zero result, the external states of the form factor must carry the opposite charge (in the all-outgoing convention). This means that the form factors involving only gluon states vanish. At three points only the form factor with two scalars, or one scalar and two fermions, are nonvanishing. Here we consider only the form factors for two external scalars and a gluon. The results are valid for any massless theory with $n_{f} \geq 1$ fermions and $n_{s} \geq 1$ scalars that has a Yukawa coupling $g_{\text {Yukawa }} \bar{\psi}^{1} \psi^{2} \phi_{12}$, and $g_{\text {Yukawa }}=g_{\mathrm{YM}}$. Then all the cuts become identical to those of $\mathcal{N}=4 \mathrm{SYM}$.

\section{The $\left(\mathcal{O}_{s}, 2_{s}^{+}, 3_{s}^{+}, 4^{+}\right)$Configuration}

For the configuration $\left(\mathcal{O}_{s}, 2_{s}^{+}, 3_{s}^{+}, 4^{+}\right)$the cut integrands are

$$
\mathcal{I}_{6}=\mathcal{I}_{1}=\mathcal{I}_{2}=0, \quad \mathcal{I}_{5}=\mathcal{I}_{3}=\mathcal{I}_{4}=\left\langle 3\left|\left(\ell_{1}+k_{4}\right)\right| 2\right]\langle 23\rangle[34]^{2}
$$

All the poles will be at most simple, and it is straightforward to obtain the residues in each case. The only nonvanishing ones are

$$
R_{\mathcal{G}_{4}}=R_{\mathcal{G}_{2}}=s_{23} s_{34} \frac{[34]\langle 23\rangle}{\langle 24\rangle}
$$

or, using vector notation,

$$
R=s_{23} s_{34} \frac{[34]\langle 23\rangle}{\langle 24\rangle}(0,1,0,1,0,0,0,0) .
$$


The integral coefficients are then given by

$$
c_{1}=P_{1} \cdot R=\frac{1}{2} s_{23} s_{34} \frac{[34]\langle 23\rangle}{\langle 24\rangle},
$$

and

$$
c_{2}=P_{2} \cdot R=-\frac{1}{2} s_{23} s_{34} \frac{[34]\langle 23\rangle}{\langle 24\rangle} \frac{1}{k_{1}^{b} \cdot k_{4}} .
$$

2. The $\left(\mathcal{O}_{s}, 2_{s}^{+}, 3^{+}, 4_{s}^{+}\right)$Configuration

The case $\left(\mathcal{O}_{s}, 2_{s}^{+}, 3^{+}, 4_{s}^{+}\right)$is related to the above configuration via the swap $k_{3} \leftrightarrow k_{4}$, $\mathcal{I}_{3} \leftrightarrow \mathcal{I}_{5}, \mathcal{I}_{1} \leftrightarrow \mathcal{I}_{6}$. The integrand is then

$$
\mathcal{I}_{6}=\mathcal{I}_{1}=\mathcal{I}_{2}=0, \quad \mathcal{I}_{5}=\mathcal{I}_{3}=\mathcal{I}_{4}=\left\langle 4\left|\left(\ell_{1}+k_{3}\right)\right| 2\right]\langle 24\rangle[43]^{2}
$$

The nonvanishing residues are again equal

$$
R_{\mathcal{G}_{4}}=R_{\mathcal{G}_{2}}=-s_{23} s_{34} \frac{[34]\langle 24\rangle}{\langle 23\rangle} ;
$$

alternatively we have

$$
R=-s_{23} s_{34} \frac{[34]\langle 24\rangle}{\langle 23\rangle}(0,1,0,1,0,0,0,0) .
$$

The integral coefficients are then given by

$$
c_{1}=P_{1} \cdot R=-\frac{1}{2} s_{23} s_{34} \frac{[34]\langle 24\rangle}{\langle 23\rangle},
$$

and

$$
c_{2}=P_{2} \cdot R=\frac{1}{2} s_{23} s_{34} \frac{[34]\langle 24\rangle}{\langle 23\rangle} \frac{1}{k_{1}^{b} \cdot k_{4}} .
$$

\section{The $\left(\mathcal{O}_{s}, 2^{+}, 3_{s}^{+}, 4_{s}^{+}\right)$Configuration}

Finally, for the configuration $\left(\mathcal{O}_{s}, 2^{+}, 3_{s}^{+}, 4_{s}^{+}\right)$, we have

$$
\mathcal{I}_{6}=\mathcal{I}_{1}=\mathcal{I}_{2}=0, \quad \mathcal{I}_{5}=\mathcal{I}_{3}=\mathcal{I}_{4}=\frac{\left\langle 3\left|\left(\ell_{1}+k_{4}\right)\right| 2\right] s_{34}^{2}}{\langle 32\rangle}
$$

The nonvanishing residues are

$$
R_{\mathcal{G}_{4}}=R_{\mathcal{G}_{2}}=s_{34}^{2} \frac{[23]\langle 34\rangle}{\langle 24\rangle}
$$


alternatively,

$$
R=s_{34}^{2} \frac{[23]\langle 34\rangle}{\langle 24\rangle}(0,1,0,1,0,0,0,0) .
$$

The integral coefficients are then given by

$$
c_{1}=P_{1} \cdot R=\frac{1}{2} s_{34}^{2} \frac{[23]\langle 34\rangle}{\langle 24\rangle},
$$

and

$$
c_{2}=P_{2} \cdot R=-\frac{1}{2} s_{34}^{2} \frac{[23]\langle 34\rangle}{\langle 24\rangle} \frac{1}{k_{1}^{b} \cdot k_{4}} .
$$

The remaining nonvanishing gluon-scalar configurations $\left(\mathcal{O}_{s}, 2_{s}^{+}, 3_{s}^{+}, 4^{-}\right),\left(\mathcal{O}_{s}, 2_{s}^{+}, 3^{-}, 4_{s}^{+}\right)$ and $\left(\mathcal{O}_{s}, 2^{-}, 3_{s}^{+}, 4_{s}^{+}\right)$can be obtained from spinor conjugations of the above ones. (The fact that the scalars are complex does not interfere with this relation, as has been explicitly checked.)

\section{Comparison to Previous Results for $\left(\mathcal{O}_{s}, 2_{s}^{+}, 3_{s}^{+}, 4^{+}\right)$}

Here we compare to the results of Brandhuber, Travaglini and Yang [85]. They obtain the following answer for the numerator of the double-box integral:

$$
N_{\mathrm{DB}}=\frac{\langle 23\rangle}{\langle 34\rangle\langle 42\rangle} s_{34}\left(s_{24} \ell_{1} \cdot k_{4}-s_{23} \ell_{1} \cdot k_{3}\right) \text {. }
$$

The integrand of the maximal cut is simply this numerator $\mathcal{I}_{i}=N_{\mathrm{DB}}$ for all kinematical solutions. Clearly, this integrand is different from the ones we computed above. However, the difference should be due to terms that integrate to zero.

Computing the residues we recognize the same expression that appeared in eq. (17.32). But this time there are more nonzero residues,

$$
R_{\mathcal{G}_{3}}=R_{\mathcal{G}_{1}}=R_{\mathcal{G}_{4}}=R_{\mathcal{G}_{2}}=\frac{1}{2} s_{23} s_{34} \frac{[34]\langle 23\rangle}{\langle 24\rangle}
$$

Two of the residues vanish due to an identity,

$$
R_{\mathcal{G}_{5}}=R_{\mathcal{G}_{6}}=\frac{1}{2} \frac{[34]\langle 23\rangle}{\langle 24\rangle}\left(2 s_{24} k_{1}^{b} \cdot k_{4}-2 s_{23} k_{1}^{b} \cdot k_{3}\right)=0 .
$$

Writing this as a vector gives

$$
R=\frac{1}{2} s_{23} s_{34} \frac{[34]\langle 23\rangle}{\langle 24\rangle}(1,1,1,1,0,0,0,0) .
$$


The integral coefficients are then given by

$$
c_{1}=P_{1} \cdot R=\frac{1}{2} s_{23} s_{34} \frac{[34]\langle 23\rangle}{\langle 24\rangle},
$$

and

$$
c_{2}=P_{2} \cdot R=-\frac{1}{2} s_{23} s_{34} \frac{[34]\langle 23\rangle}{\langle 24\rangle} \frac{1}{k_{1}^{b} \cdot k_{4}} .
$$

These results agree with the integral coefficients that we computed in section VIIB 1, so we find complete agreement with the results of ref. [85].

\section{CONCLUSIONS}

In this paper, we have continued the approach of ref. [62] in developing the maximal generalized unitarity method for two-loop amplitudes. Cutting propagators can be viewed as replacing the original real loop-momentum contours of integration by contours encircling the global poles of the integrand. Double-box integrals, independent of the configuration of external masses, have eight independent global poles. The possible ways of cutting propagators, or equivalently the allowed linear combinations of contours surrounding global poles, are constrained by the requirement that the evaluation along any contour respect the vanishing of certain insertions of Levi-Civita symbols, as well as of total derivatives arising from integration-by-parts identities. We derived the corresponding constraint equations for dou-

ble boxes with up to three external masses. The number of master integrals depends on the configuration of the external momenta, but in all cases the constraint equations yield unique and simple formulæ for the coefficients of the master integrals. The master formula (6.1), along with the projectors, given in eqs. (6.4) and (6.7), are our principal results.

\section{Acknowledgments}

KJL thanks the Institute for Advanced Study in Princeton, where part of this work was carried out, for its hospitality. We also thank Simon Caron-Huot for many helpful discussions. This work is supported by the European Research Council under Advanced Investigator Grant ERC-AdG-228301.

[1] G. Aad et al. [ATLAS Collaboration], Phys. Lett. B 716, 1 (2012) arXiv:1207.7214 [hep-ex]]. 
[2] S. Chatrchyan et al. [CMS Collaboration], arXiv:1207.7235 [hep-ex]].

[3] R. K. Ellis, I. Hinchliffe, M. Soldate and J. J. van der Bij, Nucl. Phys. B 297, 221 (1988).

[4] E. L. Berger, E. Braaten and R. D. Field, Nucl. Phys. B 239, 52 (1984).

[5] P. Aurenche, A. Douiri, R. Baier, M. Fontannaz and D. Schiff, Z. Phys. C 29, 459 (1985).

[6] Z. Bern, L. J. Dixon and C. Schmidt, Phys. Rev. D 66, 074018 (2002) arXiv:hep-ph/0206194.

[7] A. Gehrmann-De Ridder, T. Gehrmann, E. W. N. Glover and G. Heinrich, JHEP 0711, 058 (2007) arXiv:0710.0346 [hep-ph]];

S. Weinzierl, Phys. Rev. Lett. 101, 162001 (2008) arXiv:0807.3241 [hep-ph]].

[8] G. Dissertori, A. Gehrmann-De Ridder, T. Gehrmann, E. W. N. Glover, G. Heinrich, G. Luisoni and H. Stenzel, JHEP 0908, 036 (2009) arXiv:0906.3436 [hep-ph]];

G. Dissertori, A. Gehrmann-De Ridder, T. Gehrmann, E. W. N. Glover, G. Heinrich and H. Stenzel, Phys. Rev. Lett. 104, 072002 (2010) [arXiv:0910.4283 [hep-ph]].

[9] Z. Bern, L. J. Dixon, D. C. Dunbar and D. A. Kosower, Nucl. Phys. B 425, 217 (1994) hep-ph/9403226; Nucl. Phys. B 435, 59 (1995) hep-ph/9409265];

Z. Bern, L. J. Dixon and D. A. Kosower, Ann. Rev. Nucl. Part. Sci. 46, 109 (1996) hep-ph/9602280.

[10] Z. Bern and A. G. Morgan, Nucl. Phys. B 467, 479 (1996) arXiv:hep-ph/9511336.

[11] Z. Bern, L. J. Dixon and D. A. Kosower, Nucl. Phys. B 513, 3 (1998) hep-ph/9708239.

[12] Z. Bern, L. J. Dixon, D. C. Dunbar and D. A. Kosower, Phys. Lett. B 394, 105 (1997) arXiv:hep-th/9611127.

[13] R. Britto, F. Cachazo and B. Feng, Nucl. Phys. B 725, 275 (2005) hep-th/0412103.

[14] R. Britto, F. Cachazo and B. Feng, Phys. Rev. D 71, 025012 (2005) hep-th/0410179;

S. J. Bidder, N. E. J. Bjerrum-Bohr, L. J. Dixon and D. C. Dunbar, Phys. Lett. B 606, 189 (2005) hep-th/0410296;

S. J. Bidder, N. E. J. Bjerrum-Bohr, D. C. Dunbar and W. B. Perkins, Phys. Lett. B 612, 75 (2005) hep-th/0502028];

S. J. Bidder, D. C. Dunbar and W. B. Perkins, JHEP 0508, 055 (2005) hep-th/0505249;

Z. Bern, N. E. J. Bjerrum-Bohr, D. C. Dunbar and H. Ita, JHEP 0511, 027 (2005) hep-ph/0507019;

N. E. J. Bjerrum-Bohr, D. C. Dunbar and W. B. Perkins, JHEP 0804, 038 (2008) arXiv:0709.2086 [hep-ph]]. 
[15] Z. Bern, L. J. Dixon and D. A. Kosower, Phys. Rev. D 73, 065013 (2006) hep-ph/0507005.

[16] R. Britto, E. Buchbinder, F. Cachazo and B. Feng, Phys. Rev. D 72, 065012 (2005) hep-ph/0503132

R. Britto, B. Feng and P. Mastrolia, Phys. Rev. D 73, 105004 (2006) hep-ph/0602178;

P. Mastrolia, Phys. Lett. B 644, 272 (2007) hep-th/0611091].

[17] A. Brandhuber, S. McNamara, B. J. Spence and G. Travaglini, JHEP 0510, 011 (2005) hep-th/0506068.

[18] G. Ossola, C. G. Papadopoulos and R. Pittau, Nucl. Phys. B 763, 147 (2007) hep-ph/0609007.

[19] Z. Bern, L. J. Dixon and D. A. Kosower, Annals Phys. 322, 1587 (2007) [0704.2798 [hep-ph]].

[20] D. Forde, Phys. Rev. D 75, 125019 (2007) [0704.1835 [hep-ph]].

[21] S. D. Badger, JHEP 0901, 049 (2009) [0806.4600 [hep-ph]].

[22] C. Anastasiou, R. Britto, B. Feng, Z. Kunszt and P. Mastrolia, Phys. Lett. B 645, 213 (2007) [hep-ph/0609191]; JHEP 0703, 111 (2007) [hep-ph/0612277];

W. T. Giele, Z. Kunszt and K. Melnikov, JHEP 0804, 049 (2008) [arXiv:0801.2237 [hep-ph]].

[23] R. Britto and B. Feng, Phys. Rev. D 75, 105006 (2007) hep-ph/0612089. JHEP 0802, 095 (2008) [0711.4284 [hep-ph]];

R. Britto, B. Feng and P. Mastrolia, Phys. Rev. D 78, 025031 (2008) arXiv:0803.1989 [hep$\mathrm{ph}]] ;$

R. Britto, B. Feng and G. Yang, JHEP 0809, 089 (2008) [arXiv:0803.3147 [hep-ph]].

[24] C. F. Berger and D. Forde, Ann. Rev. Nucl. Part. Sci. 60, 181 (2010) arXiv:0912.3534 [hep$\mathrm{ph}]$.

[25] Z. Bern, J. J. Carrasco, T. Dennen, Y. T. Huang and H. Ita, Phys. Rev. D 83, 085022 (2011) arXiv:1010.0494 [hep-th]].

[26] R. K. Ellis, W. T. Giele and Z. Kunszt, JHEP 0803, 003 (2008) [0708.2398 [hep-ph]].

[27] C. F. Berger, Z. Bern, L. J. Dixon, F. Febres Cordero, D. Forde, H. Ita, D. A. Kosower and D. Maître, Phys. Rev. D 78, 036003 (2008) [0803.4180 [hep-ph]].

[28] G. Ossola, C. G. Papadopoulos and R. Pittau, JHEP 0803, 042 (2008) arXiv:0711.3596 [hep-ph]].

[29] P. Mastrolia, G. Ossola, C. G. Papadopoulos and R. Pittau, JHEP 0806, 030 (2008) arXiv:0803.3964 [hep-ph]]. 
[30] W. T. Giele and G. Zanderighi, JHEP 0806, 038 (2008) arXiv:0805.2152 [hep-ph]];

R. K. Ellis, W. T. Giele, Z. Kunszt, K. Melnikov and G. Zanderighi, JHEP 0901, 012 (2009) arXiv:0810.2762 [hep-ph]].

[31] C. F. Berger, Z. Bern, L. J. Dixon, F. Febres Cordero, D. Forde, T. Gleisberg, H. Ita, D. A. Kosower and D. Maître, Phys. Rev. Lett. 102, 222001 (2009) [0902.2760 [hep-ph]].

[32] G. Bevilacqua, M. Czakon, C. G. Papadopoulos, R. Pittau and M. Worek, JHEP 0909, 109 (2009) arXiv:0907.4723 [hep-ph]].

[33] P. Mastrolia, G. Ossola, T. Reiter and F. Tramontano, JHEP 1008, 080 (2010) arXiv:1006.0710 [hep-ph]].

[34] C. F. Berger et al., Phys. Rev. Lett. 106, 092001 (2011) arXiv:1009.2338 [hep-ph]].

[35] S. Badger, B. Biedermann and P. Uwer, Comput. Phys. Commun. 182, 1674 (2011) arXiv:1011.2900 [hep-ph]].

[36] V. Hirschi, R. Frederix, S. Frixione, M. V. Garzelli, F. Maltoni and R. Pittau, JHEP 1105, 044 (2011) [arXiv:1103.0621 [hep-ph]].

[37] Z. Bern, J. S. Rozowsky and B. Yan, Phys. Lett. B 401, 273 (1997) arXiv:hep-ph/9702424.

[38] C. Anastasiou, Z. Bern, L. J. Dixon and D. A. Kosower, Phys. Rev. Lett. 91, 251602 (2003) [arXiv:hep-th/0309040].

[39] Z. Bern, L. J. Dixon and V. A. Smirnov, Phys. Rev. D 72, 085001 (2005) arXiv:hep-th/0505205.

[40] Z. Bern, M. Czakon, D. A. Kosower, R. Roiban and V. A. Smirnov, Phys. Rev. Lett. 97, 181601 (2006) [arXiv:hep-th/0604074].

[41] Z. Bern, M. Czakon, L. J. Dixon, D. A. Kosower and V. A. Smirnov, Phys. Rev. D 75, 085010 (2007) arXiv:hep-th/0610248.

[42] Z. Bern, L. J. Dixon, D. A. Kosower, R. Roiban, M. Spradlin, C. Vergu and A. Volovich, Phys. Rev. D 78, 045007 (2008) arXiv:0803.1465 [hep-th]].

[43] N. Arkani-Hamed, J. L. Bourjaily, F. Cachazo, S. Caron-Huot and J. Trnka, JHEP 1101, 041 (2011) arXiv:1008.2958 [hep-th]].

[44] D. A. Kosower, R. Roiban and C. Vergu, Phys. Rev. D 83, 065018 (2011) arXiv:1009.1376 [hep-th]].

[45] N. Arkani-Hamed, J. L. Bourjaily, F. Cachazo and J. Trnka, JHEP 1206, 125 (2012) arXiv:1012.6032 [hep-th]]. 
[46] Z. Bern, C. Boucher-Veronneau and H. Johansson, Phys. Rev. D 84, 105035 (2011) arXiv:1107.1935 [hep-th]].

[47] Z. Bern, J. J. M. Carrasco, H. Johansson and D. A. Kosower, Phys. Rev. D 76, 125020 (2007) arXiv:0705.1864 [hep-th]].

[48] Z. Bern, J. J. M. Carrasco, L. J. Dixon, H. Johansson and R. Roiban, Phys. Rev. D 78, 105019 (2008) [arXiv:0808.4112 [hep-th]].

[49] F. Cachazo, arXiv:0803.1988 [hep-th];

F. Cachazo, M. Spradlin and A. Volovich, Phys. Rev. D 78, 105022 (2008) arXiv:0805.4832 [hep-th]].

[50] N. Arkani-Hamed, F. Cachazo, C. Cheung and J. Kaplan, JHEP 1003, 020 (2010) arXiv:0907.5418 [hep-th]].

[51] Z. Bern, J. J. M. Carrasco, L. J. Dixon, H. Johansson and R. Roiban, Phys. Rev. D 82, 125040 (2010) arXiv:1008.3327 [hep-th]].

[52] J. J. M. Carrasco and H. Johansson, Phys. Rev. D 85, 025006 (2012) arXiv:1106.4711 [hepth]].

[53] J. J. M. Carrasco and H. Johansson, J. Phys. A 44, 454004 (2011) arXiv:1103.3298 [hep-th]].

[54] Z. Bern, J. J. M. Carrasco, H. Johansson and R. Roiban, arXiv:1207.6666 [hep-th].

[55] Z. Bern, L. J. Dixon and D. A. Kosower, JHEP 0001, 027 (2000) arXiv:hep-ph/0001001.

[56] Z. Bern, A. De Freitas and L. J. Dixon, JHEP 0203, 018 (2002) arXiv:hep-ph/0201161.

[57] Z. Bern, A. De Freitas and L. J. Dixon, JHEP 0109, 037 (2001) arXiv:hep-ph/0109078.

[58] Z. Bern, A. De Freitas, L. J. Dixon and H. L. Wong, Phys. Rev. D 66, 085002 (2002) arXiv:hep-ph/0202271.

[59] Z. Bern, A. De Freitas and L. J. Dixon, JHEP 0306, 028 (2003) arXiv:hep-ph/0304168].

[60] Z. Bern, L. J. Dixon and D. A. Kosower, JHEP 0408, 012 (2004) arXiv:hep-ph/0404293.

[61] A. De Freitas and Z. Bern, JHEP 0409, 039 (2004) arXiv:hep-ph/0409007.

[62] D. A. Kosower and K. J. Larsen, Phys. Rev. D 85, 045017 (2012) arXiv:1108.1180 [hep-th]].

[63] P. Mastrolia and G. Ossola, JHEP 1111, 014 (2011) arXiv:1107.6041 [hep-ph]].

[64] S. Badger, H. Frellesvig and Y. Zhang, JHEP 1204, 055 (2012) arXiv:1202.2019 [hep-ph]]; JHEP 1208, 065 (2012) arXiv:1207.2976 [hep-ph]].

[65] P. Mastrolia, E. Mirabella, G. Ossola and T. Peraro, Phys. Lett. B 718, 173 (2012) arXiv:1205.7087 [hep-ph]]. 
[66] R. Kleiss, I. Malamos, C. Papadopoulos and R. Verheyen, arXiv:1206.4180 [hep-ph]].

[67] B. Feng and R. Huang, arXiv:1209.3747 [hep-ph]].

[68] P. Mastrolia, E. Mirabella, G. Ossola and T. Peraro, arXiv:1209.4319 [hep-ph]].

[69] P. Mastrolia, E. Mirabella, G. Ossola, T. Peraro and H. van Deurzen, arXiv:1209.5678 [hep$\mathrm{ph}]$.

[70] Y. Zhang, JHEP 1209, 042 (2012) [arXiv:1205.5707 [hep-ph]].

[71] S. Caron-Huot and K. J. Larsen, JHEP 1210, 026 (2012) [arXiv:1205.0801 [hep-ph]].

[72] J. Gluza, K. Kajda and D. A. Kosower, Phys. Rev. D 83, 045012 (2011) arXiv:1009.0472 [hep-th]].

[73] E. I. Buchbinder and F. Cachazo, JHEP 0511, 036 (2005) arXiv:hep-th/0506126.

[74] F. V. Tkachov, Phys. Lett. B 100, 65 (1981);

K. G. Chetyrkin and F. V. Tkachov, Nucl. Phys. B 192, 159 (1981).

[75] S. Laporta, Phys. Lett. B 504, 188 (2001) hep-ph/0102032. S. Laporta, Int. J. Mod. Phys. A 15, 5087 (2000) hep-ph/0102033.

[76] T. Gehrmann and E. Remiddi, Nucl. Phys. B 580, 485 (2000) hep-ph/9912329.

[77] R. N. Lee, JHEP 0807, 031 (2008) arXiv:0804.3008 [hep-ph]].

[78] C. Anastasiou and A. Lazopoulos, JHEP 0407, 046 (2004) hep-ph/0404258.

[79] A. V. Smirnov, JHEP 0810, 107 (2008) [0807.3243 [hep-ph]].

[80] C. Studerus, Comput. Phys. Commun. 181, 1293 (2010) arXiv:0912.2546 [physics.comp-ph]].

[81] A. V. Smirnov and A. V. Petukhov, Lett. Math. Phys. 97, 37 (2011) arXiv:1004.4199 [hep-th]].

[82] F. Wilczek, Phys. Rev. Lett. 39, 1304 (1977);

M. A. Shifman, A. I. Vainshtein and V. I. Zakharov, Phys. Lett. B 78, 443 (1978);

T. Inami, T. Kubota and Y. Okada, Z. Phys. C 18, 69 (1983).

[83] T. Gehrmann, M. Jaquier, E. W. N. Glover and A. Koukoutsakis, JHEP 1202, 056 (2012) arXiv:1112.3554 [hep-ph]].

[84] C. Duhr, JHEP 1208, 043 (2012) arXiv:1203.0454 [hep-ph]].

[85] A. Brandhuber, G. Travaglini and G. Yang, JHEP 1205, 082 (2012) arXiv:1201.4170 [hepth]].

[86] Z. Bern, J. J. M. Carrasco and H. Johansson, Phys. Rev. D 78, 085011 (2008) arXiv:0805.3993 [hep-ph]]. 